Comparative computational study of SARS-CoV-2 receptors antagonists from already approved drugs

Micael D. L. Oliveira, Kelson M. T. Oliveira

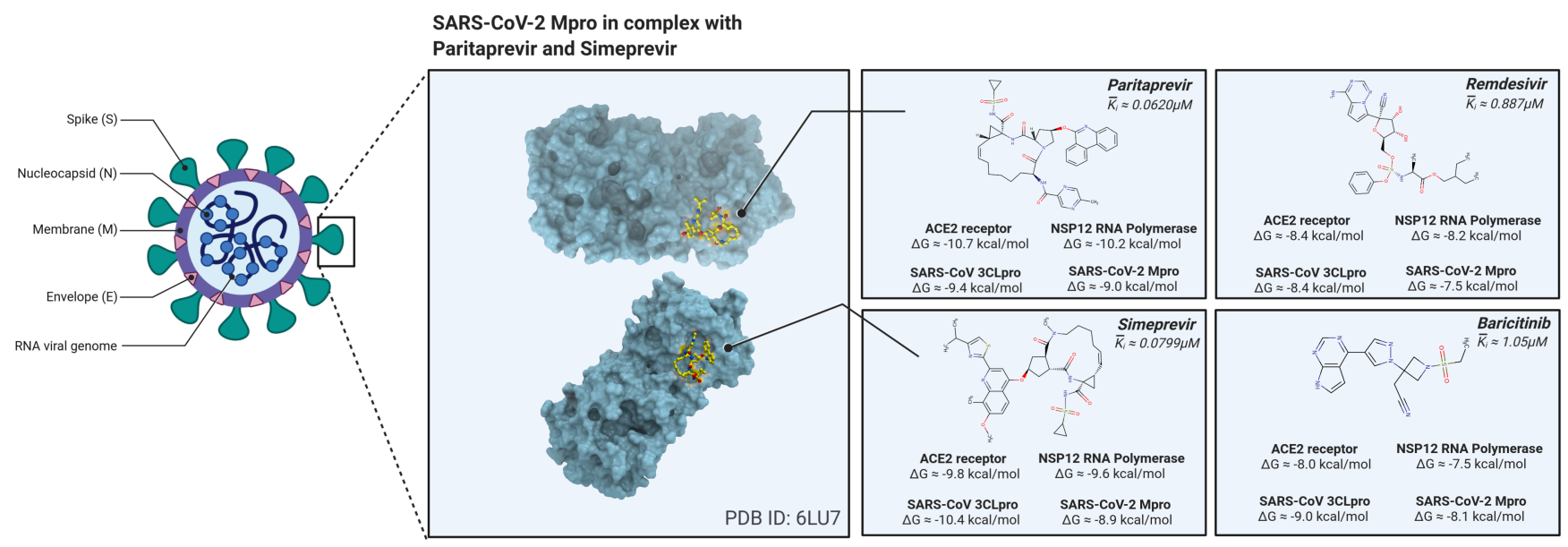

Figure: Diagram of the main results obtained in this research. The illustration was created with the aid of the BioRender.com tool. 


\section{Highlights}

Comparative computational study of SARS-CoV-2 receptors antagonists from already approved drugs

Micael D. L. Oliveira, Kelson M. T. Oliveira

- Predict antagonists of SARS-CoV-2 Mpro, SARS-CoV 3CLpro, ACE2 Receptor and NSP12 RNA Polymerase against COVID-19, based on already approved drugs, using last disease mechanisms discoveries.

- Study the affinity between ligands and the binding site through molecular docking.

- Simulate the molecular dynamics of receptor-ligand interaction to understand the potential of protein modulation and unfolding. 


\title{
Comparative computational study of SARS-CoV-2 receptors antagonists from already approved drugs
}

\author{
Micael D. L. Oliveira ${ }^{a,{ }^{* *}}, K_{\text {Kelson M. T. Oliveira }}{ }^{a *}$ \\ ${ }^{a}$ Laboratory of Theoretical and Computational Chemistry. Department of Chemistry, Federal University of Amazonas, 69077-000, \\ Manaus, AM, Brazil
}

\section{ARTICLE INFO}

\section{Keywords:}

SARS-CoV-2

ACE2 antagonists

Remdesivir

Molecular docking

Molecular dynamics

Virtual screening

ADME

\begin{abstract}
A B S T R A C T
According to the World Health Organisation, on April 12, 2020, the number of confirmed cases of COVID-19 has already exceeded an estimate of 1600000 and 105000 deaths worldwide. Given this, the impact of COVID-19 on humanity cannot be overlooked, and basic research are urgently needed. This research aims to find antagonists already approved for another diseases, that may inhibit activity of the main protease (Mpro) of the SARS-CoV-2 virus, as well as modulate the ACE2 receptors, largely found in lung cells and reduce viral replication by inhibiting NSP12 RNA Polymerase. Docking molecular simulations were realized among a total of 28 ligands published in the literature against COVID-19. Docking studies were made with algorithm of AutoDock Vina 1.1.2 software. A structure-based virtual screening was performed with MTiOpenScreen. Subsequently, the physical-chemical and pharmacokinetic parameters were analyzed with SwissADME in order to select only the most promising ones. Finally, simulations of molecular dynamics with elapsed time of 4 nanoseconds (ns) were analysed in order to better understand the action of drugs to the detriment of the limitations of molecular docking. This work has shown that, in comparative terms, simeprevir, paritaprevir and elbasvir are currently among the most theoretical promising drugs in remission of symptoms from the disease. An important structure that has already been reported in preclinical and clinical studies, in which theoretical results also corroborates high modulation in viral receptors, is the most promising among all: indinavir. The second group of promising drugs includes remdesivir, baricitinib and azithromycin, which also have clinical tests. Apparently, the repurposing drugs (hydroxy)chloroquine and chloroquine were not showed effective, as monotherapies, against SARS-CoV-2 virus or ACE2 receptors found predominantly in pneumocytes. Meanwhile, it has not been able to reach conclusive results due to the limitations of computational techniques that do not take into account numerous empirical parameters.
\end{abstract}

\section{Introduction}

The first cases of SARS-CoV-2 infections were in December, 2019, at Wuhan, Hubei province, China. Among the first 425 patients with confirmed NCIP, the mean age was 59 years and 56\% were male. The development of the epidemic follows an exponential growth. It is notable that few of the first cases occurred in children and almost half occurred in adults aged 60 or over [1].

Coronavirus ( $\mathrm{CoV}$ ) belongs to the Coronaviridae family, Nidovirales order. CoVs are divided into four genera: $\alpha-, \beta-, \gamma-$, and $\delta$ - coronavirus [2]. Unfor-

E-mail addresses: micaeloliveira@ufam.edu.br

(M.D.L. Oliveira), kelsonmota@ufam.edu.br (K.M.T. Oliveira).

*Corresponding author

** Principal corresponding author tunately, until on April 12, 2020, a total of 1696588 cases had been confirmed with 105952 deaths around 210 countries, including China [3]. Severe acute respiratory syndrome (SARS-CoV) and Middle East respiratory syndrome (MERS-CoV) are two viruses with a high propensity to transmission and pathogens that appeared in humans in the beginning of the 21st century. Both viruses possibly originated from bats [4].

The COVID-19 infection caused clusters of severe respiratory illness similar to severe acute respiratory syndrome coronavirus and was associated with ICU admission and high mortality. Major gaps in our knowledge of the origin, epidemiology, duration of human transmission, and clinical spectrum of disease still remain [5]. The onset of severe acute respiratory syndrome (SARS) was about 18 years ago, and the ge- 
netic sequences are almost identical and share $79.6 \%$ of sequence identity with SARS-CoV [6]. This similarity may prove useful in a theoretical comparative study with drugs that have shown effectiveness against SARS.

A research with a patient in the city of Munich, who during his stay, was well, with no signs or symptoms of infection. After collecting a sample from the patient, he underwent a quantitative reverse transcriptase-chain polymerase (qRT-PCR) assay showing a high viral load of $10^{8}$ copies per milliliter [7].

As the understanding of the pathogenesis of coronaviruses grows, the rational design of possible medications increases. The role of the immune system is important, and so the combination of therapies designed to simultaneously inhibit viral replication and pro-inflammatory citokines would be very promising. In addition, treatment should be started as early as possible, rather than waiting until the patient develops extensive lung damage [8].

\subsection{Clinical characteristics}

The SARS-CoV-2 beta-coronavirus, causes severe respiratory illness and has been spreading around the world rapidly. This raises an urgent need for an effective treatment of the deadly disease. However, current antiviral drugs have limited effects on virus [9].

In the literature, COVID-19 is characterised by the symptoms of viral pneumonia such as fever, fatigue, dry cough, and lymphopenia [10]. Infection with the SARSCoV-2 virus has a most probability replication in older patients with comorbidities. Most patients have mild symptoms and a good prognosis. Patients in a more serious condition affected by the virus have been developing severe pneumonia, pulmonary edema, ARDS (acute respiratory distress syndrome) or multiple organ failure [11].

\subsection{Immune responses}

When the immune response is impaired, the virus spreads and causes a generalized destruction of the affected tissues, especially organs with high expression of ACE2, causing lung inflammation. However, not all people exposed to SARS-CoV-2 are infected, and not all infected patients develop symptoms of severe acute respiratory syndrome (SARS) [12]. Using the SARS-CoV$2 \mathrm{~S}$ protein pseudovirus system, it was confirmed that the human angiotensin-converting enzyme 2 (hACE2) is an important SARS-CoV-2 receptor found predominantly in 293T cells in lung tissue [13].

\subsection{Molecular mechanisms of SARS-CoV-2}

Coronaviridae polyproteins are cleaved and transformed in mature non-structural proteins (NSPs) by the two proteases 3CLpro (3C-like protease) and PLpro (Papain Like Protease) [14]. The coronaviruse genome encodes 4 structural proteins: spike glycoprotein (S), small envelope protein (E), matrix glycoprotein (M) and nucleocapsid protein $(\mathrm{N})$. In addition, the 3CLpro, a main protease required for the maturation of coronaviruses, is vital for the viral cycle, making it an important target, and generating hoping to thousands of people [15].

An important fact about SARS Coronavirus is activated by a trypsin-like protease, largely present in the lung, appears essential to activate the virus for cell binding [16]. SARS spike glycoprotein have two cleavage sites known for that virus. They are assumed to be the same cleavage points with high degree of homology in comparison with SARS-CoV-2 [17]. To date, there is still no specific antiviral therapy for the SARS-CoV2 virus, and the main treatments are only to alleviate symptoms. Recombinant IFN with ribavirin has limited effects against $\mathrm{CoV}$ infection. After SARS and Epidemics of MERS, great efforts were devoted to the development of new antivirals targeting $\mathrm{CoV}$ proteases, 
polymerases, MTases and entry proteins, however, none of them have been shown to be effective in clinical trials [18].

The $\mathrm{CoV}$ spike $(\mathrm{S})$ glycoprotein is a key target for vaccines, therapeutic antibodies, and diagnostics. The atomic-level details enable the design and screening of small molecules with fusion-inhibiting potential [19]. Coronavirus spike(S) glycoproteins promote entry into cells and are the main target of antibodies. SARSCoV-2 (S) glycoprotein uses ACE2 to enter cells. The cross-neutralizing antibodies targeting conserved S epitopes can be important targets for future vaccines [20]. In this context, the expression of the ACE2 receptor is found in many extrapulmonary tissues including heart, kidney, endothelium, and intestine. Suggesting that the ACE2-expressing AECII facilitate coronaviral replication in the lung. Thus, ACE2 could important target this interaction site with antibodies or small molecules [21]. The SARS-CoV-2 virus uses the cell entry receptor Angiotensin II Converting Enzyme (ACE2), the same used by SARS-CoV. The virus genome consists of six large open reading structures (ORFs) common to other coronaviruses [6]. In this sense, ACE2 binding is a critical determinant for the host range of SARS-CoV. The SARS-CoV-2 virus uses the angiotensin-converting enzyme $2(\mathrm{ACE} 2)$ as a receptor and mainly infects bronchial hair epithelial cells and type II pneumocytes, while MERS-CoV uses dipeptidyl peptidase 4 (DDP4) [4]. ACE2, is an ACE homologous. The common ACE isoform is also present in other vascularized tissues, including heart, brain, striated muscle and kidney, and is not restricted to endothelial cells [22].

\subsection{Receptors involved in virus RNA replication}

There are several functional coronavirus proteins involved in transcription, translation, synthesis, processing and modification of RNA, virus replication and infection. Among these, 3CLpro, PLpro, RdRp and helicase are the most important targets for the development of inhibitors [23]. PLpro is responsible for cleaving the $\mathrm{N}$-terminus of the replicase polyprotein to release Nsp1, Nsp2 and Nsp3, which are essential for virus replication [24]. Constituting a central enzyme in the coronavirus replication process, PLpro has been a popular target for coronavirus inhibitors [23]. 3CLpro (chymotrypsin-like protease), also known as Nsp5, is first automatically cleaved from polyproteins to produce mature enzymes and then further cleaves Nsps downstream at 11 sites to release Nsp4-Nsp16. 3CLpro directly mediates the maturation of the Nsps, essential in the viral cycle [25]. 3CLpro and PLpro are two proteases that process the polypeptide translation product of genomic RNA into the structural and nonstructural protein vital to viral replication. RdRp acts in the polymerization of viral RNA having a deep cavity as an active site [26]. Nsp12 is an RNA-dependent RNA polymerase ( $\mathrm{RdRp})$ and a vital coronavirus replication / transcription complex enzyme [27]. In principle, targeted inhibition of Nsp12-RdRp would have significantly reduced toxicity and side effects in host cells, but no specific inhibitors have been found so far [23].

\subsection{Promissing compounds}

For the development of medicines treating SARSCoV-2, the fastest way is to find potential molecules from the marketed drugs [23]. Another research discovered that carfilzomib, eravacycline, valrubicin, elbasvir, streptomycin are very promissing to have affinity of interaction with the receptor SARS-Cov-2 [28]. A recently research concluded that paritaprevir and simeprevir, were identified as potential inhibitors of SARS-CoV-2 3CLpro [29]. At the Rajavithi Hospital in Thailand, the infectious disease team used a combination of oseltamivir (anti-influenza agent) and lopinavir 
plus ritonavir (HIV protease inhibitors) to successfully improve patients with severe conditions [30]. Remdesivir was also suggested to be potential inhibitor against SARS-CoV-2 [26].

Many promising researches are conducting theoretical tests with anti-virals [15]. Another importants ligands are: Atazanavir, nelfinavir, amprenavir, fosamprenavir, remdesivir [30]. In mice, both prophylactic and therapeutic remdesivir improve pulmonary function and reduce lung viral loads and severe lung pathology [31]. Other finding reveal that chloroquine is highly effective in the control of SARS-CoV-2 infection in vitro [32]. Camostat increased the survival rate of mice infected with SARS-CoV [26].

A very recent study demonstrated the clinical characteristics of 135 patients with COVID-19, who received treatment with Kaletra (lopinavir/ritonavir) and traditional Chinese medicine, having an important contribution to the treatment of viral pneumonia. [33]

Clinical trial with $600 \mathrm{mg}$ (hydroxy)chloroquine daily is associated with the reduction, or even, disappearance of viral load, in patients affected by COVID-19, with its effect enhanced when administering azithromycin as an adjuvant. It was suggested a synergistic effect between both [34]. Pre-clinical studies have indicated that remdesivir can effectively reduce the viral load in the lung tissue of mice infected with the MERS-CoV virus, improving lung function and damage to lung tissue [35]. Elfiky et al. reports that drugs like sofosbuvir, ribavirin, and remdesivir used like anti-HCV, nucleotide inhibitors, can be repurposing against the new strain of coronavirus that emerged with promising results [36]. After sequencing of SARS-CoV-2, researches suggested a few compounds that are likely to be effective, including the antiretroviral lopinavir plus ritonavir combination [37]. Baricitinib was predicted to reduce the ability of the virus to infect lung cells, being a potential treatment for COVID-19 acute respiratory disease
$[38]$.

\section{Methods and materials}

It is very important to find a medication that has efficacy and safety for the patient. Many antivirals violate the Lipinski's Rule, contributing to greater difficulty in absorption and elimination by oral administration. These concerns are essential, because patients affected by COVID-19 have a very weak immune response, potentiating some medications to even worsen symptoms. The main purpose of this research was analyze the existing drugs that are more thermodynamically effective in inhibiting the main receptors involved in viral replication. To this end, a series of theoretical methodologies were used, such as homology analysis, molecular docking and molecular dynamics, in order to quickly identify among the best drugs suggested at the moment, the most effective.

\subsection{Crystallographic structures}

Using the Protein Data Bank (PDB) database, the crystallographic structures of target proteins were obtained. In searches where more than one result appeared, priority was given to articles published in 2020 and higher resolutions. The two characterization methods found correspond to Electron Microscopy and Xrays diffraction. All protein visualisations were through Molsoft ICM-Browser software.

The crystalographic structure of main protease SARSCoV-2 used in this work presenting a high resolution of $2.16 \AA$, containing a total of 306 residues and approximately $31.4 k D a$ of molecular mass (see Figure 1). This crystal structure was originally resolved by Liu et al. from diffraction detector source through synchrotron radiation at SSRF (Shanghai Synchrotron Radiation Facility) Beamline BL17U1 with crystallized protein at $p H \approx 6[39]$. 


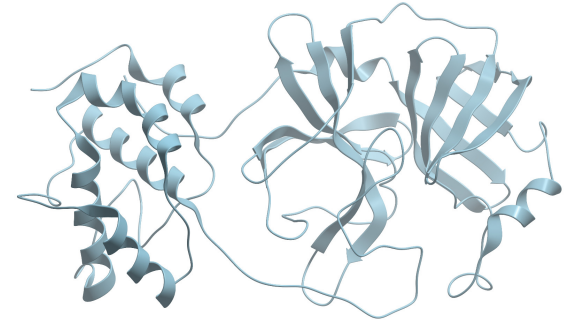

Figure 1: The crystal structure of COVID-19 main protease with PDB ID: 6LU7 [39]

In its turn, the protein SARS-CoV 3CLpro (see Figure 2) has a resolution of $2.50 \AA$ with a total of 603 residues (chains $\mathrm{A}$ and $\mathrm{B}$ ) and approximately $62.0 \mathrm{kDa}$ of molecular mass [40]. The complex of SARS-CoV-2 spike with ACE2 receptor (Figure 3) has a resolution of $2.45 \AA$ with a total of 791 residues(chains $\mathrm{A}$ and $\mathrm{E}$ ) and a molecular mass near of $84.8 k \mathrm{Da}$ [41].

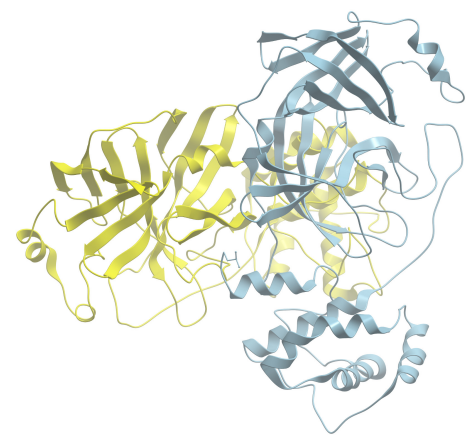

Figure 2: Crystal structure of SARS Coronavirus Main Proteinase (3CLpro) with PDB ID: $1 U K 4$ [40]

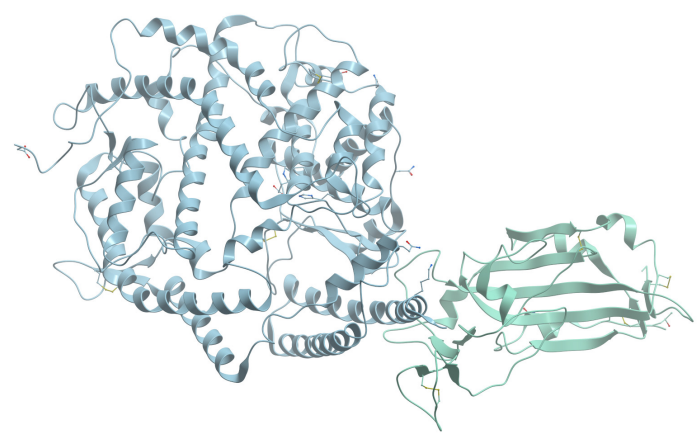

Figure 3: Crystal structure of 2019-nCoV spike receptorbinding domain bound with ACE2 with PDB ID: 6MOJ [41]

The complex of SARS-Coronavirus NSP12 and NSP7, NSP8 co-factors used an Electron Microscopy to determine the structure (Figure 4). Has a resolution of $3.10 \AA$ with a total of 1435 residues(chains A, B, C and D) and a molecular mass near of $114.54 k \mathrm{Da}$ [42].

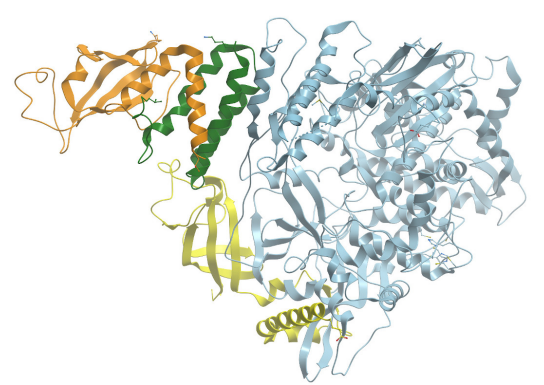

Figure 4: SARS-Coronavirus NSP12 bound to NSP7 and NSP8 co-factors with PDB ID: 6NUR [42]

Initially, the amino acid sequence was downloaded from $\mathrm{PDB}$ repository in the extension .fasta. Subsequently, through this sequence there was a search for correspondents classified by an alignment score using the HMM-HMM correspondence method and subsequent confidence calculation. Confidence represents the percentage probability (from 0 to 100 ) that the correspondence between the sequence and the prediction is a true homology. Being $>90 \%$, it means that the protein adopts the general fold and that the core of the protein is modeled with high precision $(2-4 \AA$ RMSD of the true and native structure). The percentage identity indicates the probable precision of the model between the provided sequence and the possible homology [43]. Thus, the homology result using the Phyre2 tool at "Intensive" modelling mode, showed a similarity of the SARS-CoV-2 main protease to the Trypsin-like serine proteases superfamily (Figure 5). Confidence of homology obtained was near $100 \%$ with accuracy $>90 \%$ [43]. This indicates that drugs that can affect structures similar to the Trypsin-like serine proteases superfamily can also favorably affect SARS-CoV-2 to some degree. 


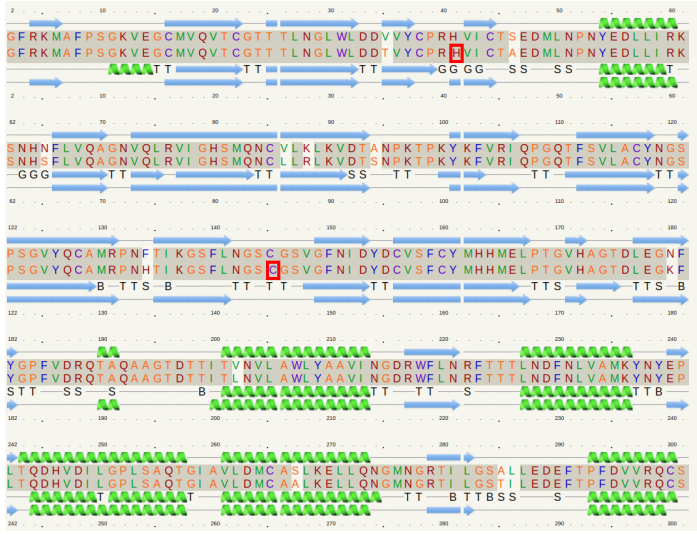

Figure 5: Alignment of amino acids sequences between protease of SARS-CoV-2 and Trypsin-like serine proteases superfamily.

\subsection{Structural validation of proteins}

In principle, dihedral angles $\phi$ and $\psi$ in amino acids can have any value between $+180^{\circ}$ and $-180^{\circ}$, but several values are prohibited by steric impediment between the atoms of the polypeptide skeleton and the side chains of the amino acids. To validate whether the proteins skeleton conformation is the best possible, the use of a Ramachandran diagram is very useful, once test the quality of three-dimensional structures of proteins [44]. Therefore, validation of proteins under investigation was confirmed by the RAMPAGE tool (Ramachandran Plot Investigation) and can be see in the (Figure 6). Protein structure validation criteria are routinely applied during the deposition of structures to the databases. For good structural quality, it is recommended to have over $98.0 \%$ of residues in the favored region [45]. After submitting the.$p d b$ files, the SARS-CoV-2 Mpro structure demonstrated that 97.4\% of the residues are present in a favored region. Although, SARS-CoV 3CLpro protein presented $91.0 \%$ of amino acids in a favorable region, both have excellent structural quality. The same is true for the ACE2 receptor with $97.6 \%$ in a favorable region, and for the NSP12 RNA Polymerase with $98.7 \%$ of the residues.
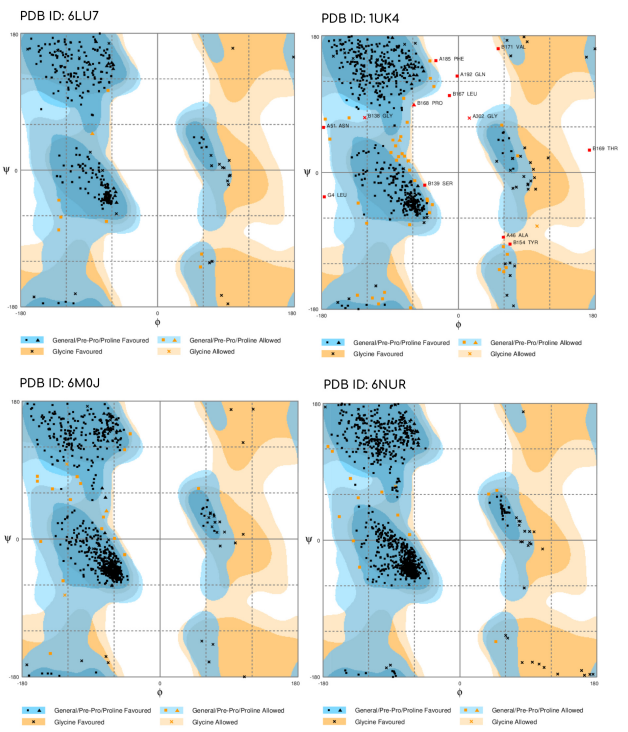

PDB ID: GNUR

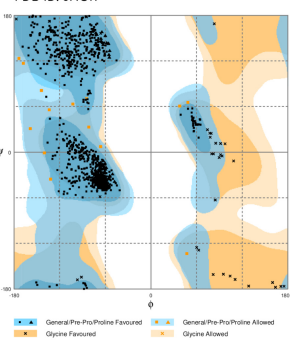

Figure 6: Comparison of structural validation between SARSCoV-2 Mpro (PDB ID: 6LU7), SARS-CoV 3CLpro (PDB ID: 1UK4), ACE2 Receptor (PDB ID: 6M0J) and NSP12 RNA Polymerase (PDB ID: 6NUR) receptors by the Ramanchandran map.

\subsection{Structure-Based Virtual Screening}

Through the MTiOpenScreen tool, there was done be a virtual screening of drugs already approved by the pharmaceutical industry. The filters used was based on the Lipinski's Rule for oral bioavailability (as seen below) [46]. In this context, the analysis of octanol/water partition coefficient $(\log P)$ is a vital property to study lipophilicity and reduce the risk of toxicity [47].

Lipophilicity: $-0,7<x \log P 3<+5,0$

Size: $150 \mathrm{~g} / \mathrm{mol}<M W<500 \mathrm{~g} / \mathrm{mol}$

Polarity: $20 \AA^{2}<T P S A<130 \AA^{2}$

Insolubility $0<\log S(E S O L)<6$

Insaturation: 0, $25<\mathrm{Csp} 3$ (fractionary) $<1$

Flexibility: $0<$ Rotatable bonds $<9$

The effectiveness and safety of a medication depends essentially on the biotransformations that occur in the organism [48]. In the development of medications, virtual screening can be performed on filters of large pharmaceutical companies, among which: the 
Lipinski's filter (Pfizer), the Ghose (Amgen), Veber (GSK), Egan (Pharmacia) and Muegge (Bayer) [46]. However, many medications are approved even though they violate some parameters of these filters. Thus, ADME results are not a rule, but they are important to have a theoretical idea of the pharmacological action of a drug.

\subsection{Prediction of binding sites}

Main binding site was calculated by blind-docking using a search algorithm in AutoDock Vina 1.1.2 on Linux $x 64$ bits architecture. The docking calculations in AutoDock Vina consist in a number of sequential steps, that involves a random perturbation of the conformation followed by a local optimization and a posterior selection in which a determined step can be accepted $[49,50]$. Thus, the prediction was done according to the coordinates of the conformation of smaller free energy. The RMSD cut-off value of $2.00 \AA$ is often used as a criterion for the validity of the docking results [51]. All the visualisation was through the Molsoft ICM-Browser, are presented in (Figure 7).

All predictions of main binding site were made with complex of (hydroxy)chloroquine. The main bindingsite of protease SARS-CoV-2 Mpro centered on ligand has absolute coordinates of grid box with: $x=$ $-11.734 ; y=12.582 ; z=67.382$. The main bindingsite of SARS-CoV 3CLpro has coordinates of grid box with: $x=63.834 ; y=-22.369 ; z=18.851$. The ACE2 receptor protein binding site has more probability to have the following coordinates of binding site: $x=$ $-21.626 ; y=34.686 ; z=-15.468$. The NSP12 RNA Polymerase enzyme has its binding site of most affinity in coordinates: $x=-15.468 ; y=144.233 ; z=126.383$. In the Figure 7, the (hydroxy)chloroquine ligand was inserted into the most likely binding site for each receptor, according to the coordinates listed above. It is very important to note that each ligand has a binding site of greater affinity. Thus, if the simulations were carried out with another ligand, the coordinates could probably be different.

\subsection{Preparation of ligands}

Initially, all ligands conformers were downloaded from the ChemSpider database in format .mol. The ligands were previously prepared using UCSF Chimera 1.14 [52] and ADT Tools 1.5.7rc1[53]. Polar hydrogens were added to the $p H \approx 7.0$, residues reached a state of protonation in these conditions of $p H$, and non-polar were merged. Gasteiger-Kollman partial charges were added to all ligands [54]. Finally, a file in the .pdb format was generated to be read in the ADT to be converted later into .pdbqt.

\subsection{Preparation of receptors}

Gasteiger-Marsili partial charges were estimated to the protein, water molecules were removed, ligands and cofactors were also be excluded. Polar hydrogens atoms were added though UCSF Chimera 1.14. Protonation state was for Histidine.

\subsection{Parameters of molecular docking}

In the development of AutoDock Vina, stochastic approaches to global optimization were explored, including Lamarckian genetic algorithms(LGA), in search of a local minimum [49] that uses a empirical-knowledge scoring function. In the score algorithms, for two atoms $i, j$, the atomic energy in pairs is evaluated by the sum of Van der Waals interactions, hydrogen bonds, Coulomb energy and desolvation [55]. Molecular docking softwares runs a search algorithm in which the conformation of the ligand is evaluated recursively until the convergence to the minimum energy is achieved. Finally, an affinity scoring function, $\Delta G[\mathrm{kcal} / \mathrm{mol}]$, a state variable, is used to classify the most favorable orientations [56]. In the analysis of the validity of the docking re- 
sults, the $R M S D$ value to the maximum of $2 \AA$ is recommended between the best conformer and the initial structure [51]. The inhibition constant is expressed by $K_{i} \equiv K_{d} \equiv e^{\Delta G / R T}$. The $R T$ product in docking simulations is about to $0.592 \mathrm{kcal} / \mathrm{mol}(T \approx 298.15 \mathrm{~K}$ and $\left.R \approx 1.987 \mathrm{kcal} . \mathrm{K}^{-1} \cdot \mathrm{mol}^{-1}\right)$. Protein stability is achieved through a native conformation, that is, with the lowest values of free energy $\Delta G$, causing greater spontaneity in the chemical reaction. Furthermore, the most likely region for receptor-ligand interactions is found at the binding site [44]. In the receptor-ligand interaction, there is an increase in order and a consequent loss of entropy [57].

The exhaustiveness parameter was the default value of 8 , that means 250000 runs. The total number of conformers to be generated was 10, with parameter energy range $=1$. To guarantee reproducibility, the pseudo ramdom-seed was adopted as 0 in all simulations. The receptor was adopted as a rigid body. The grid box size in all simulations was $126.0 \AA \times 126.0 \AA \times 126.0 \AA$, each one centered on respective mass center of receptor. Thus, the gridbox occupied the entire macromolecule centered on the following coordinates for the SARSCoV-2 Mpro receptor: $x=-26.295 ; y=12.604 ; z=$ 58.941. For the SARS-CoV 3CLpro receptor were: $x=$ 63.220; $y=-14.971 ; z=21.822$. For the ACE2 receptor were: $x=-26.895 ; y=18.454 ; z=-14.038$. Finally, for NSP12 RNA Polymerase enzyme were: $x=$ $150.009 ; y=147.554 ; z=157.012$. In the simulations in which the first conformer was adopted, the input structure was superimposed with the one obtained through docking, using the AutoDock Tools software. The matcher method was "AtomName", and therefore, without additional symmetrization, it is equivalent to RMSD u.b.(Upperacids present in the enzyme. HOMO orbitals express Bound). The conformations resulting from docking into .pdbqt were converted to the .pdb format by the OpenBabel 3.0[58] tool, in order to later visualize the interaction of amino acids and study molecular dynamics.
The computational architecture of all docking simulations, was the Ubuntu 20.04 Linux x64 bits operational system, Intel(R) Core(R) i3 6300 CPUs @ (4 cores $\times 3.80 \mathrm{GHz}$ ) and $8 \mathrm{~GB}$ RAM Memory $2133 \mathrm{MHz}$ DDR5. The docking simulations were with a total of 4 receptors: the main protease (Mpro) of the SARS-CoV2 virus with PDB ID: 6LU7; chymotrypsin-like protease (3CLpro) from the SARS-CoV virus with (PDB ID: 1UK4); the SARS-CoV-2 virus ACE2 receptor with (PDB ID: 6M0J); and RNA-dependent RNA polymerase (RdRp) NSP12 with (PDB ID: 6NUR).

\subsection{Molecular descriptors}

After docking results, the most important drugs were have their structures optimized with GAMESS (US)[59] at version June 2019 R1 software package. Due to the computational limits, the mechanical-quantum calculations of structural optimization and study of electronic descriptors, although were at the DFT level, but with less precise basis set functions, adopting the $3-21 \mathrm{G}$ gaussians with B3LYP exchange and correlation hybrid function, multiplicity equal to 1 (singlet), RHF orbital model, and treating the structure in the gas phase, disregarding the presence of a solvent. Eletrostatic potential map was created with 64000 grid points and hexadecapole accuracy with density isosurface equal a $\rho \approx 0.003 e / b o h r^{3}$. Molecular orbitals were created with a isosurface value of $\rho \approx 0.050 e / b o h r^{3}$. All visualisations were through WebMO Demo Server 19.0.009e [60]. Electronic molecular descriptors that correspond mainly to the energies of the frontier orbitals HOMO, $H O M O-1, L U M O$ and $L U M O+1$, can indicate the regions of possible interactions between orbitals and amino the regions with the greatest electrophilic potential. While the LUMO orbitals, the nucleophilic potential. 
PDB ID: 6LU7

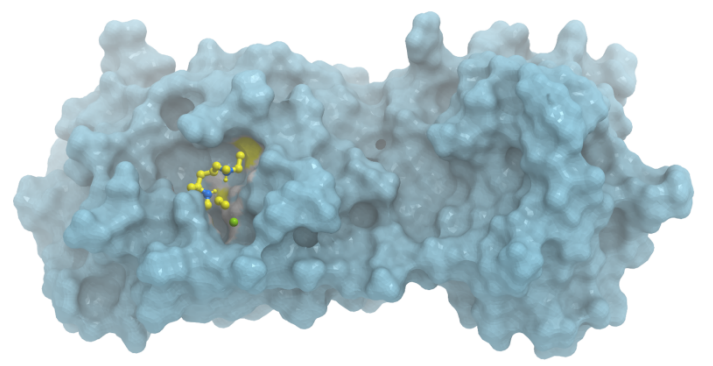

PDB ID: 6MOJ

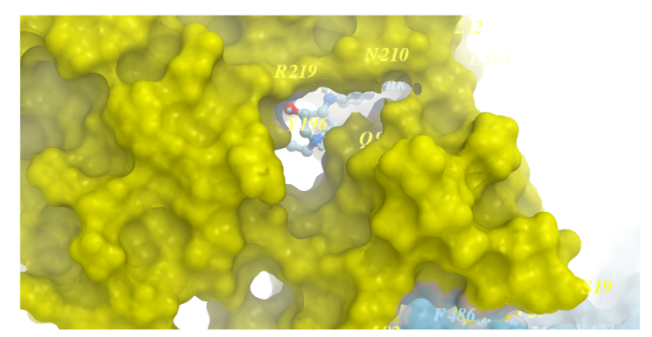

PDB ID: 1UK4

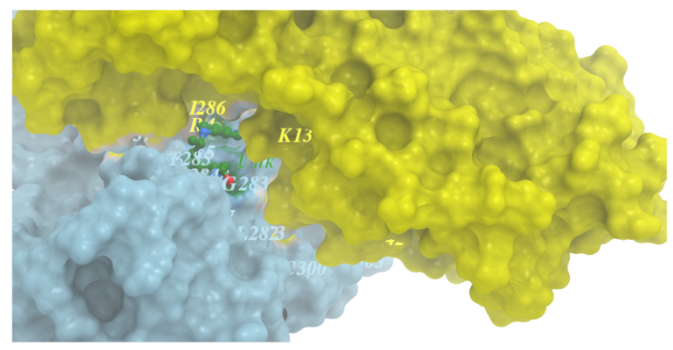

PDB ID: 6NUR

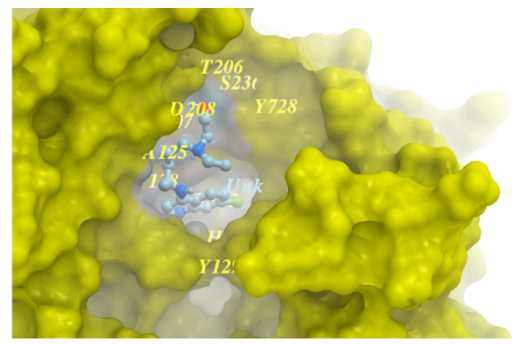

Figure 7: Visualisation of the (hydroxy)chloroquine ligand in the cavity (binding site) of most probability in each one of the receptors: SARS-CoV-2 Mpro (6LU7), SARS-CoV 3CLpro (1UK4), ACE2 Receptor (6MOJ) and NSP12 RNA Polymerase (6NUR).

\subsection{Parameters of molecular dynamics}

In molecular dynamics, successive iterations are generated by the integration of Newton's laws of motion. The result is a trajectory that specifies how the positions and velocities of particles in the system vary over time. In more realistic models, the force acting on the particle depends on its position in relation to the others, making the analytical description extremely complex [61].

The best conformers (lowest free energy and RMSD) calculated from docking simulation were used for molecular dynamics. Through the LARMD tool (Ligand and Receptor Molecular Dynamics)[62], molecular dynamics analysis were performed, considering the influence of water molecules, in the time interval equivalent to 4ns. The force field used was AMBER16. The Sander module in the $A M B E R 16$ program was used to perform the minimization in four steps before the MD simulation [63]. In all minimization processes, the 2000 steps steepest descent method and 3000 steps conjugated gradient method were used. The system was heated from 10 to $300 \mathrm{~K}$ in $30 \mathrm{ps}$. The following release process was similar to the minimization. Finally, all the atoms were relaxed in $300 \mathrm{~K}$ and $1 \mathrm{~atm}$ by applying periodic boundary conditions [62]. The objective was understand how the drugs affect the macromolecule unfolding, and therefore, the Radius of Gyration (Rg) value. The normalized RMSD of atomic displacement over time was also done analyzed.

RMSD values, in addition to indicating conformational changes, also express rotational and translational movements of the ligand when interacting with the binding site of receptor. The RMSF values express the flexibility of each receptor residue during the receptorligand interaction. In this way, it seeks to show the interaction with the amino acids of the binding site, in which the fluctuation is greater in proportion to the inhibition potential [64, 28]. 


\section{Results and Discussion}

\subsection{Molecular docking studies}

It is very important to note that the structures have not been optimized, and therefore, are not in their minimum energy state. However, this does not compromise the results obtained, since geometric optimization by the methods of molecular quantum mechanics results in structures relaxed in a vacuum (which differs from the molecular environment of docking) [65]. In addition, the receptor was considered to be a rigid body. It is most appropriate for all simulations to be carried out with an exhaustiveness of 400 , but unfortunately due to the short time available it was not be possible, thus was used the 8 default value. These considerations can have quantitatively some effect the results obtained, but not qualitatively $[66,67]$.

Due to the high degree of rotational freedom of ligands, it was found that some RMSD values exceeded the recommended of $2.00 \AA$. Thus, it was arbitrarily adopted that the free energy of the best conformer will be that which has an RMSD u.b (upper bound) at the maximum of $3.00 \AA$. In the analysis of the docking results, was opted for the first conformation with RMSD of at most $2.50 \AA$. If, among the 10 conformations generated, all exceed $2.50 \AA$, the one with the lowest RMSD will be chosen. The results of molecular docking with the main and promising drugs used in treatment against SARS-CoV-2 are shown in (Table 1). For a correct interpretation of these results, priority was given to ligands with greater potential of inhibition of ACE2 receptors.

Among all ligands, the most promising are used in the treatment of patients affected by Hepatitis-C. Simeprevir and paritaprevir, have been shown to have a higher inhibition potential than other drugs by acting as an effective antagonist in the various stages of viral replication. Only with the ACE2 receptor did simeprevir not have the best affinity value, but its inhibitory capacity is greater than drugs, like ribavirin, (hydroxy)chloroquine and chloroquine. However, medications like remdesivir, elbasvir, nelfinavir and saquinavir have also been shown to be good ACE2 receptor inhibitors. This receptor, in principle, can hinder coronaviral replication in lung cells [20]. These results corroborating with others studies that suggest to there are great and potentials drugs for partial remission of patients affected by COVID-19. In this context, an important randomized, controlled and open study with the drugs lopinavir and ritonavir in adult hospitalized patients in severe condition showed that there are no benefits beyond the standard conditions in patients affected by COVID-19 [68]. Thus, the theoretical results partially corroborate the experimental results obtained about lopinavir and ritovanir, witch presented small binding affinities than compared with simeprevir, paritaprevir and others. This is because lopinavir had a free energy of $-7.7 \mathrm{kcal} / \mathrm{mol}$ in its potential to inhibit ACE2 receptors. But its potential to act as an antagonist of the main protease of the SARS-CoV-2 virus proved relatively low of $-7.0 \mathrm{kcal} / \mathrm{mol}$, when compared to other much more promising drugs. Ritonavir shows similar results, but differently, it has a greater potential for interaction with the SARS-CoV 3CLpro protein, but reduced activity at $\mathrm{ACE} 2$ receptors.

Based on these results, it is possible to conclude that the drugs that act as antagonists of the SARS-CoV-2 Mpro receptor, responsible for the entry of the virus in human cells, also become inhibitors of related receptors, such as 3CLpro that acts in the viral cycle, or even, ACE2 receptors, the main responsible for the entry of the virus in lung cells. In addition to NSP12, vital in viral transcription and replication. Indicating that all these receptors are in fact correlated with the replication mechanism of the SARS-CoV-2 virus. Something noteworthy: the RMSD values to the baricitinib ligand 
tended to zero, because in the preparation of the structure, the degree of torsional freedom was 0, (Table 2). However, this structure has a total of 5 rotatable bonds, what partially explain your high affinity binding values. Another way to understand this affinity is to express the docking values using the inhibition constant $K_{i}$. The $K_{i}$ values are lower as the receptor-ligand interaction affinity becomes greater and and are qualitatively similar to the $I C_{50}$ values. Naturally the $I C_{50}$ values can only be obtained by experimental studies, since it is dependent on the concentration of the proteinligand complex, but the correlation can be useful (see Table 3). However, this is only an average estimate of inhibition, as it is not possible to say that all receptors have equal contributions to viral replication. Once again, $K_{i}$ values point to paritaprevir and simeprevir as the best antagonists. Paritaprevir has a very low equilibrium constant of $0.0620 \mu M$, that is, a small amount of this antagonist is sufficient to maintain a stable conformation at the receptor site and, therefore, a better inhibition rate. In comparison, chloroquine and (hydroxy)chloroquine showed high $K_{i}$ values, with the lowest score values. Chloroquine showed $K_{i} 496$ times greater than paritaprevir, and (hydroxy)chloroquine $K_{i}$ 286 times greater than simeprevir, and 22 times greater than azithromycin. These values suggest chloroquine and (hydroxy)chloroquine will only show great effectiveness at high dosages, could be present a high degree of hepatotoxicity or others adverse effects.

Through docking simulations, it can be seen that the compounds chloroquine and (hydroxy)chloroquine are theoretically shown to act as partially effective antagonists of the receptors involved in viral replication (3CLpro). It is notorious that, in contrast to some preclinical research, in particular chloroquine, appears to be effective in limiting the replication of SARS-CoV-2 in vitro [69]. However, it seems that the docking results imply only median modulation of the receivers, due to thermodynamic factors. In this context, (hydroxy)chloroquine or its similars, acting as the only antagonist, does not prove to be a reasonable candidate to direct interact with virus receptors. However, the mechanism of action of aminoquinolines, in fact, is to increase the intracellular $\mathrm{pH}$ which will decrease the probability of the virus entering the plasma membrane and interacting with ACE2 receptors. Other drugs studied in this work, based on a molecular docking model, show greater potential. Azithromycin, which is being tested as adjuvant with (hydroxy)chloroquine, in turn presented a reasonable binding energies, being a good candidate against main receptors of SARS-CoV-2 replication [34].

\subsection{Analyse of electronic descriptors}

Molecular descriptors were calculated through GAMESS (US) [59] package, and from the .out file, descriptors could be viewed were through WebMO Demo server [60]. The Molecular Electrostatic Potential Surface (MEPS) is a plot of electrostatic potential mapped onto the constant electron density surface and has been used primarily for predicting sites and relative reactivity towards electrophilic attack and in studies of biological recognition. MAPS plots express the charge distribution profile of molecules, contributing to the recognition of the likely sites of ligand-receptor interactions (Figure 8). The electronic density is obtained in computational energy optimization calculations, and then the potential surface is generated. The negative electrostatic potential corresponds to an attraction of the proton by the concentrated electron density in the molecule (colored in shades of red), the positive electrostatic potential corresponds to repulsion of the proton by atomic nuclei in regions where low electron density exists and the nuclear charge is not completely shielded (colored in shades of blue) [70]. Regions of intermediate potential, follow the color order of the spectrum: red $<$ orange $<$ 
yellow $<$ green $<$ blue. The MEPS for simeprevir indicate few regions with high positive potentials, however, in general terms, it has positive electrostatic potential, except where oxygen atoms are present, which paritaprevir has a similar behavior. Remdesivir, in turn, presents in its MAPS regions with a strong negative character potential, especially over the region of the pentacyclic ring. Because baricitinib has a small molecular structure when compared to other drugs, it has a better charge distribution, with negative potentials on oxygen atoms.

The energy gap between the HOMO (Highest Occupied Molecular Orbital) and LUMO (Lowest Unoccupied Molecular Orbital) is very important descriptor in characterization of molecular activity. With GAP is possible determining electrical properties, optical polarizability and chemical reactivity descriptors, such as hardness, softness and chemical potential, of a molecule. FMO (Frontier Molecular Orbitals) attempts to predict relative reactivity based on properties of the reactants. It is commonly formulated in terms of perturbation theory [71]. HOMO orbitals tend to concentrate predominantly close to aromatic groups, a functional group capable of performing interactions dipoledipole type, aromatic-hydrophobic and ion-dipole, having a high probability of interacting with amino acids present in the periphery of the receptor [70]. Similars energy differences between HOMO and LUMO orbitals, reflects the structural similarity between both ligands where absolute values of $\Delta E_{\text {simeprevir }} \approx 7.687 \mathrm{eV}$ e $\Delta E_{\text {paritaprevir }} \approx 8.258 \mathrm{eV}$. Thus, it can be said that simeprevir is a little more reactive compared to paritaprevir, because the reactivity is increased as the GAP decreases. However, (hydroxy)chloroquine is more reactive than both, presenting $\Delta E_{(\text {hydroxy }) \text { chloroquine }} \approx 7.165 \mathrm{eV}$. Despite contrasting the docking results, in which simeprevir and paritaprevir have greater interaction affinity, it is important to note that reactivity does not necessar- ily express direct correlation to affinity with the binding site. In highlight, there is the drug azithromicin with the highest reactivity among all drugs, it has $\Delta E_{\text {azithromicin }} \approx$ $3.862 \mathrm{eV}$. However, it may have a greater recurrence of adverse effects. The energy values of the frontier orbitals of 6 main ligands are in (Figure 9).

The reaction of a nucleophile involves the addition of electrons to the reagent, that is, the interaction of the nucleophile $H O M O$ with the reagent $L U M O$. If there is more than one possible center of attack, the preferred reaction mode is expected to occur in the atom with the largest LUMO orbital. Likewise, the reaction of an electrophile will involve the $H O M O$ of the reagent, which in the reaction should preferably occur in the atom with the largest $H O M O$ orbital [71]. The contribution of the $L U M O$ molecular orbital, represents the region of greater electro-receptor character and is mainly concentrated in the pharmacophore region for all molecules. 

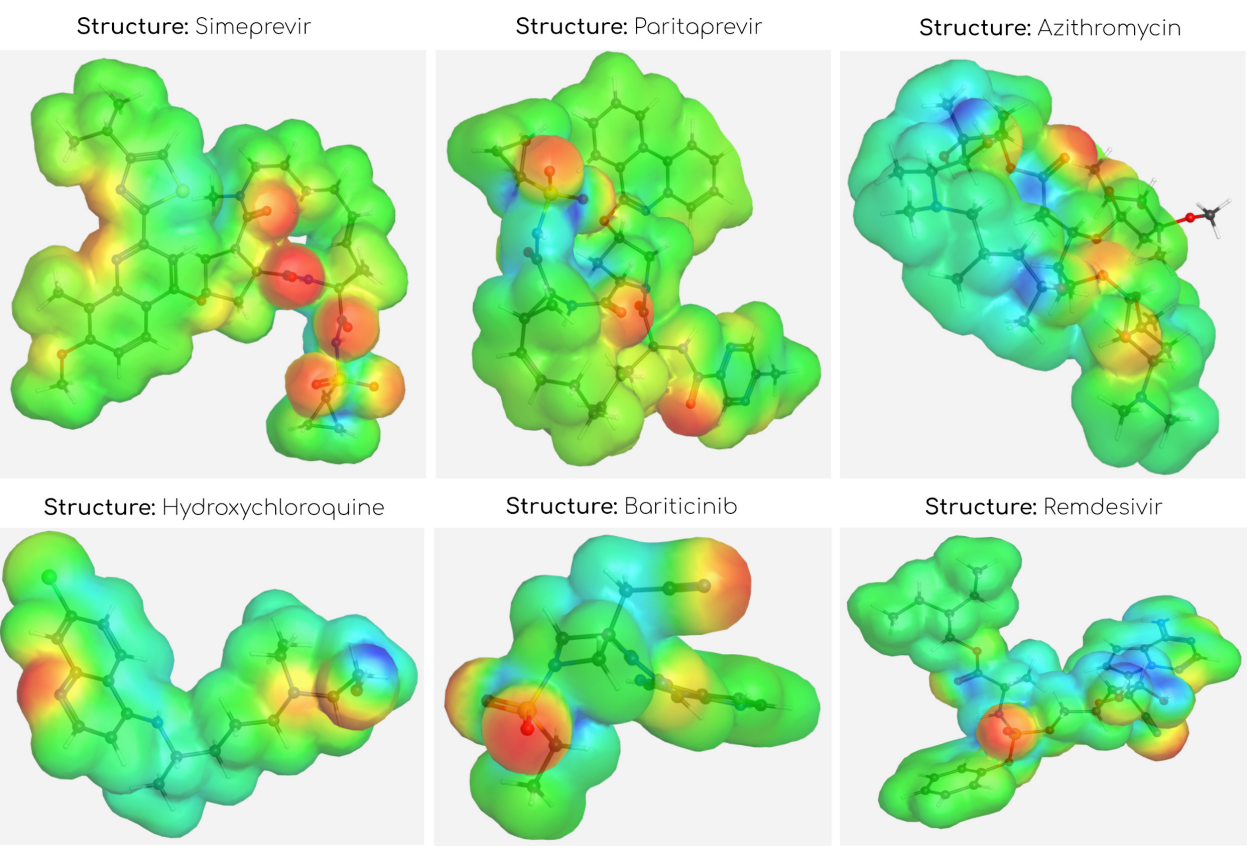

Figure 8: Eletrostatic potential maps(MEPS) of main ligands. Images are not scaled proportionally.

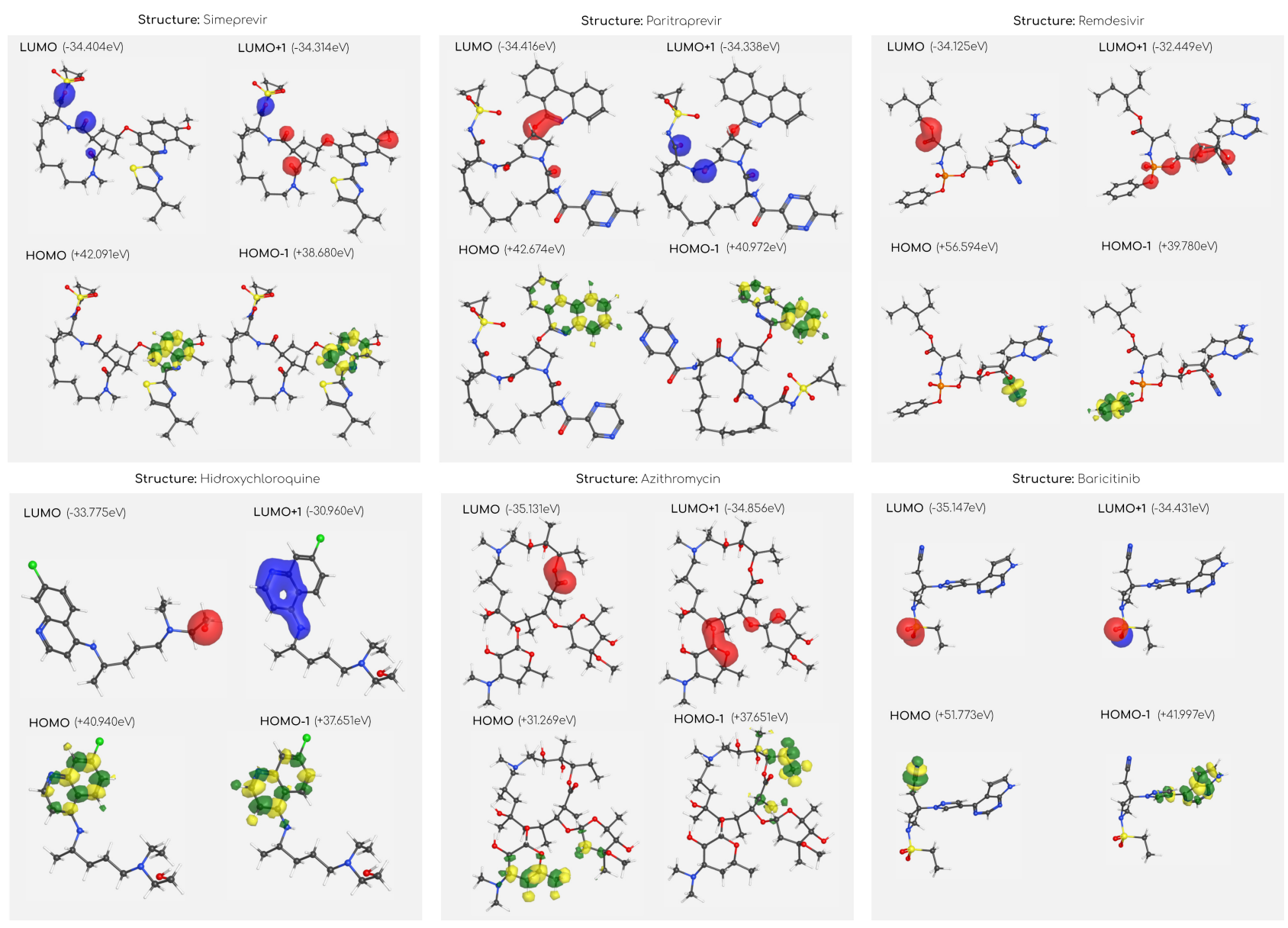

Figure 9: Visualisation of frontier molecular orbitals (FMO) of 6 main ligands. Images are not scaled proportionally. 
Table 1

Calculated binding energies $(\mathrm{kcal} / \mathrm{mol})$ for already approved drugs docked with the proteins SARS-CoV-2 Mpro, SARS-CoV 3CLpro, ACE2 Receptor and NSP12 RNA Polymerase. Ligands were organized in descending order of average Vina scores.

\begin{tabular}{|c|c|c|c|c|c|}
\hline Antagonist & SARS-CoV-2 Mpro & SARS-CoV 3CLpro & ACE2 Receptor & NSP12 RNA Polymerase & $\overline{\Delta G}$ \\
\hline Paritaprevir & -9.0 & -9.4 & -10.7 & -10.2 & -9.825 \\
\hline Simeprevir & -8.9 & -10.4 & -9.8 & -9.6 & -9.675 \\
\hline Indinavir & -7.8 & -10.5 & -10.1 & -9.5 & -9.475 \\
\hline Elbasvir & -8.6 & -10.7 & -9.1 & -8.6 & -9.250 \\
\hline Saquinavir & -7.4 & -10.3 & -8.9 & -7.9 & -8.625 \\
\hline Nelfinavir & -7.6 & -9.7 & -9.2 & -7.5 & -8.500 \\
\hline Azithromycin & -7.5 & -8.4 & -8.8 & -8.3 & -8.250 \\
\hline Baricitinib & -8.1 & -9.0 & -8.0 & -7.5 & -8.150 \\
\hline Remdesivir & -7.5 & -8.4 & -8.4 & -8.2 & -8.125 \\
\hline Sofosbuvir & -7.5 & -9.2 & -8.6 & -7.1 & -8.100 \\
\hline Eravacycline & -7.2 & -9.1 & -8.4 & -7.7 & -8.100 \\
\hline Lopinavir & -7.0 & -9.4 & -7.7 & -8.3 & -8.100 \\
\hline Ritonavir & -6.7 & -9.5 & -8.5 & -7.4 & -8.025 \\
\hline Valrubicin & -7.5 & -8.7 & -8.2 & -7.4 & -7.950 \\
\hline Camostat & -7.3 & -8.4 & -8.2 & -7.5 & -7.850 \\
\hline Tipranavir & -6.8 & -9.4 & -7.4 & -7.1 & -7.675 \\
\hline Fosamprenavir & -7.5 & -8.1 & -7.4 & -7.5 & -7.625 \\
\hline Atazanavir & -7.4 & -7.9 & -7.8 & -6.8 & -7.475 \\
\hline Carfilzomib & -6.8 & -8.5 & -7.1 & -7.2 & -7.400 \\
\hline Amprenavir & -6.5 & -8.2 & -7.4 & -7.5 & -7.400 \\
\hline Streptomycin & -6.1 & -8.1 & -7.3 & -7.6 & -7.275 \\
\hline Darunavir & -6.5 & -7.1 & -8.1 & -6.8 & -7.125 \\
\hline Umifenovir & -6.7 & -7.2 & -6.9 & -6.6 & -6.850 \\
\hline Ribavirin & -6.2 & -6.8 & -6.6 & -6.2 & -6.450 \\
\hline (Hydroxy)chloroquine & -6.0 & -6.8 & -6.1 & -6.4 & -6.325 \\
\hline Chloroquine & -5.9 & -6.4 & -6.0 & -6.3 & -6.150 \\
\hline Favipiravir & -5.7 & -5.9 & -6.1 & -5.7 & -5.850 \\
\hline Chloroquine phosphate & -3.8 & -4.1 & -3.9 & -4.5 & -4.075 \\
\hline
\end{tabular}

Table 2

Respectives RMSD (Upper Bound) values of docking simulations.

\begin{tabular}{|c|c|c|c|c|}
\hline Antagonist & SARS-CoV-2 Mpro & SARS-CoV 3CLpro & ACE2 Receptor & NSP12 RNA Polymerase \\
\hline Paritaprevir & $2.018 \AA$ & $2.021 \AA$ & $0.746 \AA$ & $2.002 \AA$ \\
\hline Simeprevir & $1.462 \AA$ & $2.652 \AA$ & $1.142 \AA$ & $1.295 \AA$ \\
\hline Baricitinib & $0.001 \AA$ & $0.001 \AA$ & $0.001 \AA$ & $0.001 \AA$ \\
\hline Remdesivir & $1.808 \AA$ & $1.887 \AA$ & $1.551 \AA$ & $2.179 \AA$ \\
\hline Azithromycin & $1.527 \AA$ & $0.640 \AA$ & $1.695 \AA$ & $2.712 \AA$ \\
\hline (Hydroxy)chloroquine & $2.035 \AA$ & $1.038 \AA$ & $1.105 \AA$ & $2.115 \AA$ \\
\hline Chloroquine & $0.783 \AA$ & $0.839 \AA$ & $2.246 \AA$ & $1.490 \AA$ \\
\hline
\end{tabular}

Table 3

Inhibition constant $\left(K_{i}\right)$ based on average Vina score from all 4 receptors.

\begin{tabular}{lll}
\hline Antagonist & $\overline{K_{i}}$ & Vina score $(\mathrm{kcal} / \mathrm{mol})$ \\
\hline Paritaprevir & $0.0620 \mu M$ & 9.825 \\
Simeprevir & $0.0799 \mu M$ & 9.675 \\
Azithromycin & $1.006 \mu M$ & 8.175 \\
Baricitinib & $1.050 \mu M$ & 8.150 \\
Remdesivir & $1.100 \mu M$ & 8.125 \\
(Hydroxy)chloroquine & $22.9 \mu M$ & 6.325 \\
Chloroquine & $30.8 \mu M$ & 6.150 \\
\hline
\end{tabular}

\subsection{Amino acids interactions}

The interaction arrangement between different molecules in a complex, provides important information about the function of proteins and the role of ligands in molecular biology. The amino acids that appear in the diagram (Figure 10) are those that participate in the pattern of interaction between ligand and receptor with an energetic contribution to the total energy of interaction of at least $33 \%$ of the maximum possible energy for this type of interaction [72]. All results were made with ProteinPlus Tools [72]. 
M.D.L Oliveira, K.M.T Oliveira / ChemRxiv (2020)
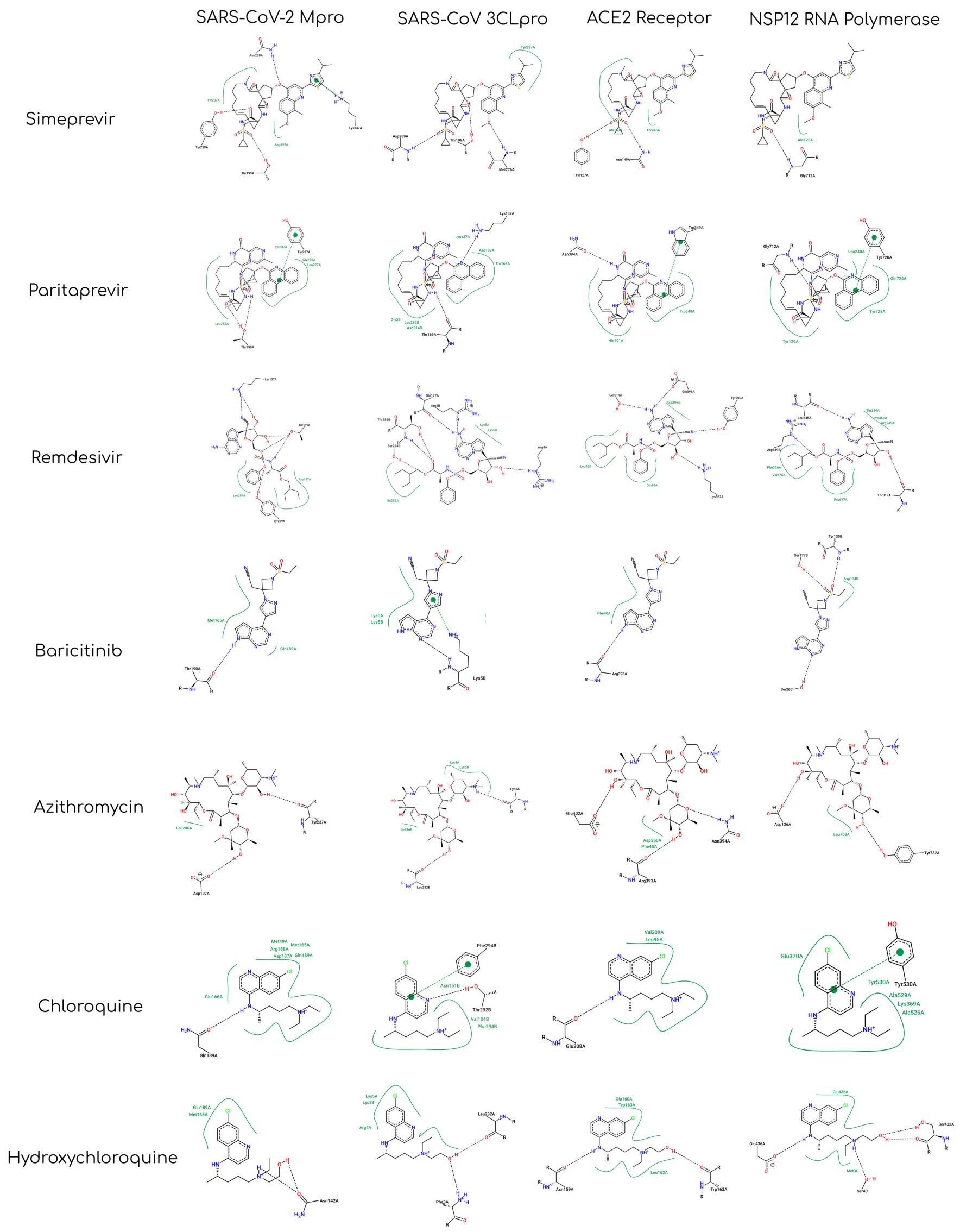

Figure 10: Diagrams of amino acids interactions for potential inhibitors binding. 
The ligands with the lowest free energy, and those most reported in the scientific literature in clinical and pre-clinical studies for the treatment of COVID-19, were presented the interaction of amino acids (Figure 10) with the respective ligand in a cutoff distance of $\approx$ $2.80 \AA$.

The drugs with the best binding affinities were also the ones that showed the greatest interactions in the form of hydrogen bonds. This shows a good inhibitory capacity, when interacting quasi-covalent form with the amino acids of the binding sites. Antagonists with low affinity values, such as chloroquine and (hydroxy)chloroquine, presented in large part non-covalent interactions in their aromatic structure, with few hydrogen bonds (usually in the chain). An exception was the interaction of (hydroxy)chloroquine with the NSP12 RNA Polymerase, which showed four interactions of hydrogen in its side chain, however with a binding affinity of $-6.4 \mathrm{kcal} / \mathrm{mol}$. The results in (Figure 11) seems to suggest that the best antagonists are those with strong hydrogen bonds together with a large amount of non-covalent interaction.

\subsection{Virtual Screening with others already approved drugs}

Virtual Screening was performed from a virtual library with 7173 ligands, most of all, already approved by pharmaceutic industry, using MTiOpenScreen [73] web server. The target receptor was the main protease of SARS-CoV-2 with PBD ID: $6 L U \%$. Initially, the structures were ordered by the lowest values of free energy values, and therefore a likely better binding affinity, without taking into account pharmacokinetic, physical-chemical properties or toxicity. Ahead, there was done a new screening selecting only those that comply with pharmaceutical filters, and that have potential for oral bioavailability. These measures were taken, in order that patients may have a higher degree of toler- ance to medications. Despite the concern as profiles for oral administration, it appears that most antagonists of the SARS-CoV-2 virus are more effective in other routes of administration. The most promising results were organized in Table 4 containing a total of 10 ligands already approved for the treatment of other diseases. It is worth mentioning the Lumacaftor, adopted in patients with Cystic Fibrosis, a pathology that also severely affects lung cells. 
Table 4

The virtual screening of possible antagonist drugs of main protease of SARS-CoV-2 (PDB ID: 6LU7), using the MTi Open Screen based on AutoDock Vina simulations.

\begin{tabular}{|c|c|c|c|}
\hline Name & Chemical Structure & Binding Energy(Vina Score) & Disease Treatment \\
\hline Fosaprepitant & & $-9.7 \mathrm{kcal} / \mathrm{mol}$ & Prevention of nausea and vomiting \\
\hline Ubrogepant & & $-9.7 \mathrm{kcal} / \mathrm{mol}$ & Acute treatment of migraine \\
\hline Ledipasvir & & $-9.4 \mathrm{kcal} / \mathrm{mol}$ & Hepatitis C \\
\hline Dihydroergotamine & & $-9.1 \mathrm{kcal} / \mathrm{mol}$ & Acute treatment of migraine \\
\hline Proscillaridin & & $-9.1 \mathrm{kcal} / \mathrm{mol}$ & Congestive heart failure \\
\hline Venetoclax & & $-9.0 \mathrm{kcal} / \mathrm{mol}$ & Chronic lymphocytic leukemia \\
\hline Etoposide Phosphate & & $-9.0 \mathrm{kcal} / \mathrm{mol}$ & Antineoplastic \\
\hline Ergotamine & & $-8.8 \mathrm{kcal} / \mathrm{mol}$ & Acute migraine attacks \\
\hline Aprepitant & & $-8.7 \mathrm{kcal} / \mathrm{mol}$ & Prevent induced nausea and vomiting \\
\hline Lumacaftor & & $-8.5 \mathrm{kcal} / \mathrm{mol}$ & Cystic Fibrosis (CF) \\
\hline
\end{tabular}




\subsection{Interatomic interactions in protein}

\section{structures}

(a1) SARS-Cov-2 in complex with Chloroquine

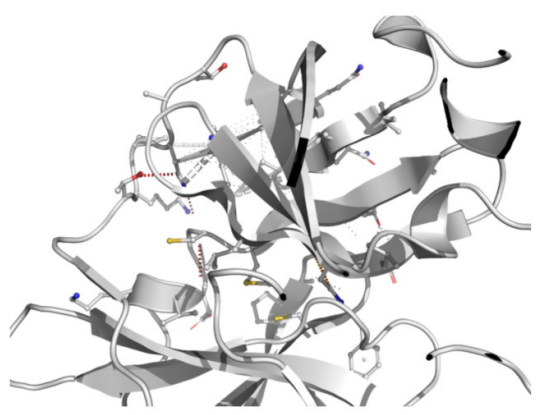

(b1) SARS-Cov-2 in complex with Remdesivir

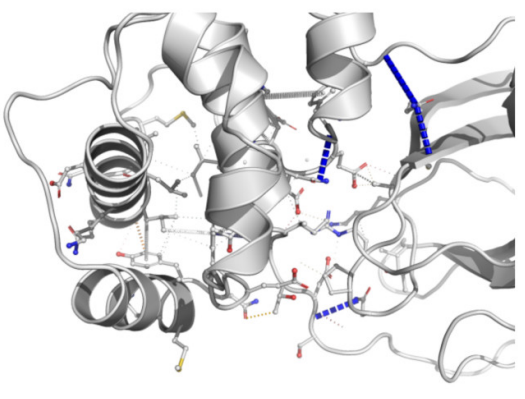

(c1) SARS-Cov-2 in complex with Simeprevir

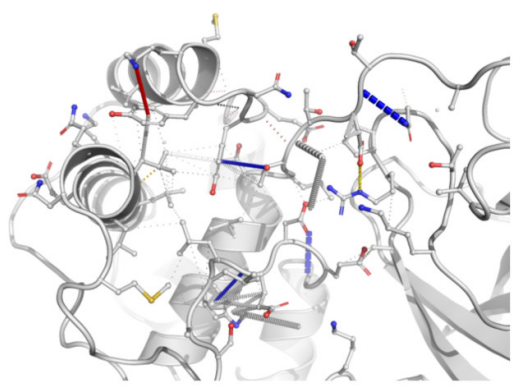

(d1) SARS-Cov-2 in complex with Paritaprevir

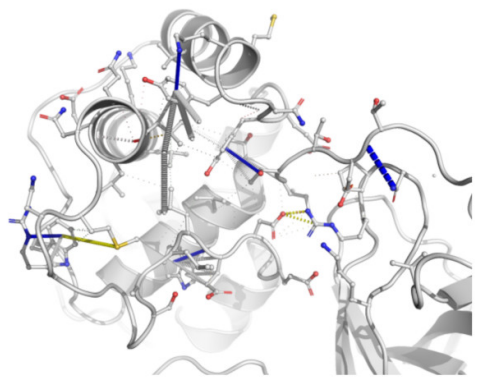

(a2) SARS-Cov 3CLpro in complex with Chloroquine

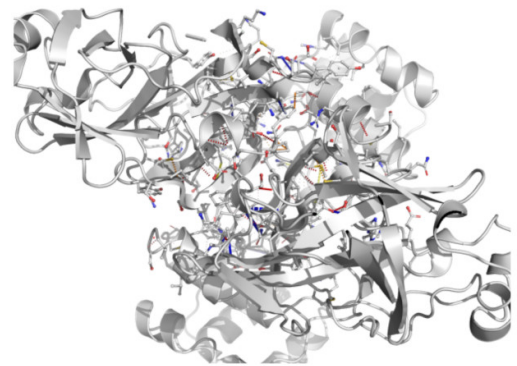

(b2) SARS-Cov 3CLpro in complex with Remdesivir

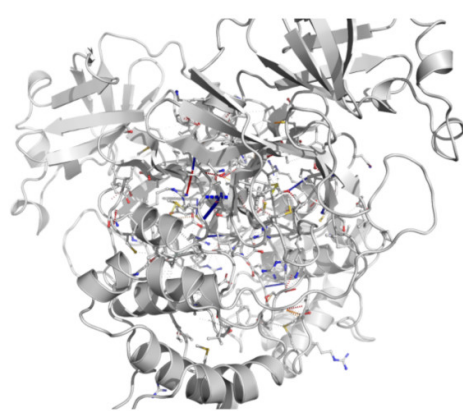

(c2) SARS-Cov 3CLpro in complex with Simeprevir

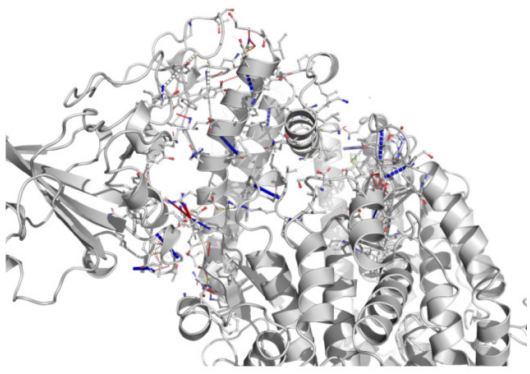

(b3) ACE2 in complex with Remdesivir
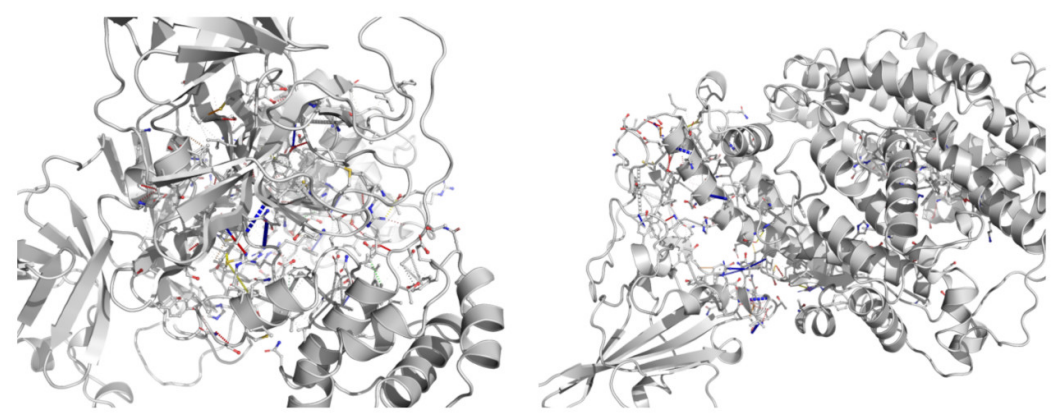

(d2) SARS-Cov 3CLpro in complex with Paritaprevir

(d3) ACE2 in complex with Paritaprevir

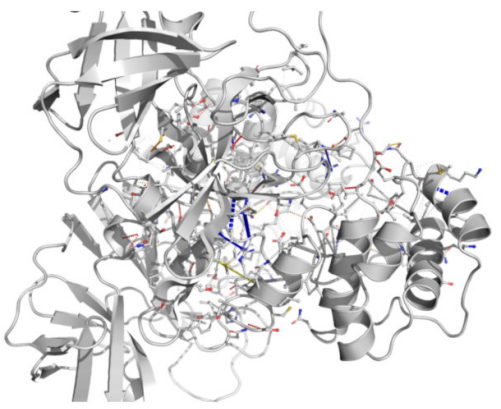

Figure 11: Visualisations of molecular interactions between the binding site and the respective drug. Main Interactions between amino acids and ligands: Van der Waals (Gray), ionic (Yellow), carbonyl (Pink), hydrophobic contacts (Green), hydrogen bonds (Red). All images were generated by the Arpeggio tool. 


\section{Table 5}

ADME parameters prediction. ${ }^{1}$ Potential for oral administration. ${ }^{2}$ Greater efficiency in other types of administration. * Individual results, requiring more in-depth analysis.

\begin{tabular}{|c|c|c|c|c|c|}
\hline Antagonist & Molar Mass $(\mathrm{g} / \mathrm{mol})$ & Rotatable Bonds & Lipophilicity $(x \log P 3)$ & $\operatorname{TPSA}\left(\AA^{2}\right)$ & Fraction Csp3 \\
\hline Simeprevir ${ }^{2}$ & 749.94 & 9 & 4.81 & 193.51 & 0.55 \\
\hline Paritaprevir ${ }^{2}$ & 765.88 & 9 & 4.65 & 198.03 & 0.42 \\
\hline Remdesivir $^{2}$ & 602.58 & 14 & 1.91 & 213.36 & 0.48 \\
\hline Baricitinib ${ }^{1}$ & 371.42 & 5 & -0.46 & 128.94 & 0.38 \\
\hline Fosaprepitant $1, *$ & 614.41 & 9 & 1.02 & 139.72 & 0.39 \\
\hline Ubrogepant $1, *$ & 549.54 & 6 & 3.11 & 104.29 & 0.34 \\
\hline Ledipasvir $2, *$ & 889.00 & 16 & 7.36 & 174.64 & 0.47 \\
\hline Dihydroergotamine ${ }^{2, *}$ & 583.68 & 5 & 2.37 & 118.21 & 0.48 \\
\hline Proscillaridin $2, *$ & 530.65 & 3 & 2.48 & 129.59 & 0.77 \\
\hline Venetoclax $2, *$ & 868.44 & 14 & 8.18 & 183.09 & 0.38 \\
\hline Etoposide Phosphate ${ }^{2, *}$ & 668.54 & 7 & -0.71 & 217.17 & 0.55 \\
\hline Ergotamine ${ }^{1, *}$ & 581.66 & 5 & 3.06 & 118.21 & 0.42 \\
\hline Aprepitant $1, *$ & 534.43 & 8 & 4.20 & 83.24 & 0.39 \\
\hline Lumacaftor ${ }^{1, *}$ & 452.41 & 6 & 4.44 & 97.75 & 0.21 \\
\hline
\end{tabular}

Most of all interactions are showed between amino acids and ligands, including Van der Waals interactions (Gray), ionic (Yellow), carbonyl (Pink), hydrophobic contacts (Green), hydrogen bonds (Red) and specific atom-aromatic ring (cation- $\pi$, donor- $\pi$, halogen- $\pi$, and carbon- $\pi$ ) and aromatic ring-aromatic ring $(\pi-\pi)$ (see Figure 11). Knowledge of the specific interactions made in macromolecular binding sites can provide insights into understanding molecular recognition. Could help understand molecular genetics mutations and drug resistance [74]. Hydrogen bonds between the ligand and receptor amino acids plays an important role in the stability of the inhibitor-protein complex, which is in accordance with the interactions between amino acids already shown in (Figure 10) [75].

\subsection{ADME prediction}

The ligands with the greatest potential for inhibition among the main receptors involved in viral replication, were analyzed for their toxicity properties and bioavailability potential for oral administration through SwissADME [46].

The medications simeprevir and paritaprevir for having a molecular mass above the recommended for oral administration, that is, values greater than $500 \mathrm{~g} / \mathrm{mol}$, in addition to having a relatively high degree of polarity (TPSA), oral administration could theoretically hinder the metabolic biotransformations of the structure and your subsequent absorption (Table 5). In addition, reduced expression of ACE2 is associated with hypertension and defects in cardiac contractility [48].

It is important to thoroughly analyze the two most promising medications, simeprevir and paritaprevir. The SwissADME tool predicted that simeprevir inhibits cytochrome P450 3A4 that acts on oxidation and subsequent elimination by the organism. It is noticed that it does not inhibit other cytochromes of the P450 family, indicating a low gastrointestinal absorption. In addition to little water-soluble, when presenting solubility of $5.44 \times 10^{-5} \mathrm{mg} / \mathrm{mL}$ on the $\log S$ scale $(E S O L)$. The prediction of paritaprevir also indicates that it inhibits cytochrome $P 450344$, and therefore also has low gastroinstestinal absorption. Because they have similar structures, it is also poorly water-soluble with a solubility of $4.91 \times 10^{-5} \mathrm{mg} / \mathrm{mL}$ calculated by the topological method Log $S$ (ESOL). In contrast, the drug (hydroxy)chloroquine interacts with cytochromes CYP2D6 and CYP1A2, presenting a high gastrointestinal absorption. Presenting potential for retinal toxicity, neuromyopathy and heart disease [76]. 
3.7. Molecular dynamics studies

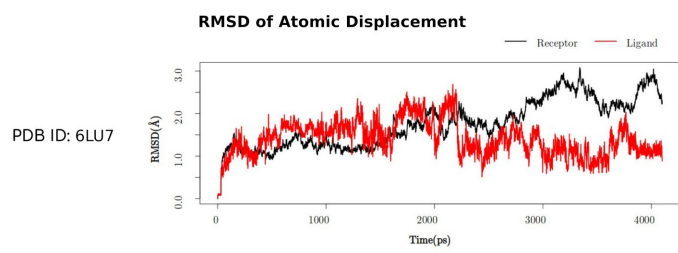

RMSF of atomic positional fluctuation
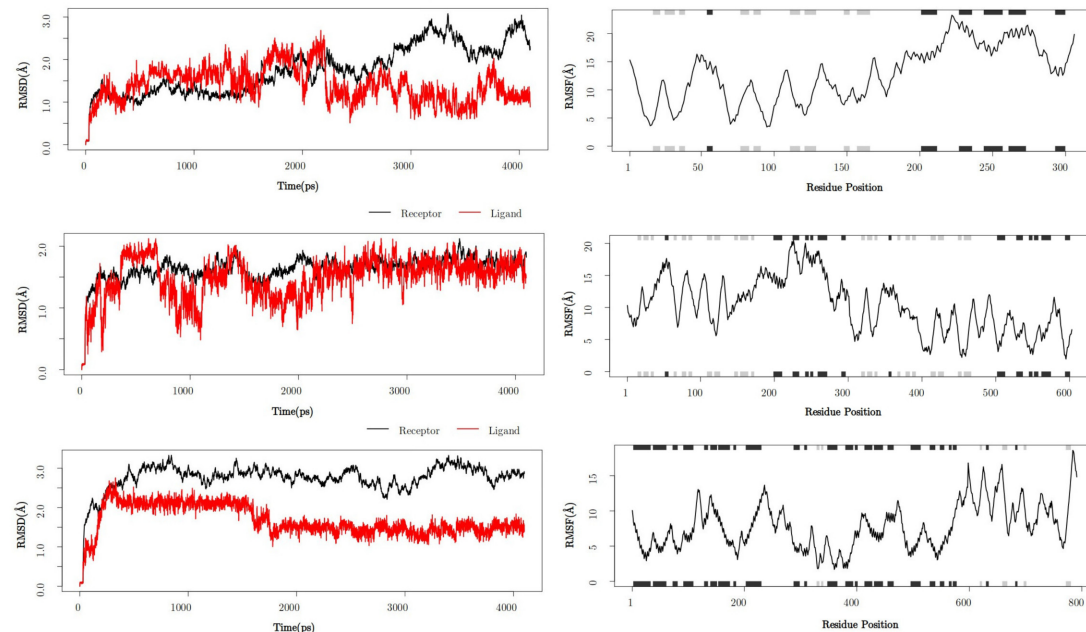

Radius of Gyration $(\mathbf{R g})$
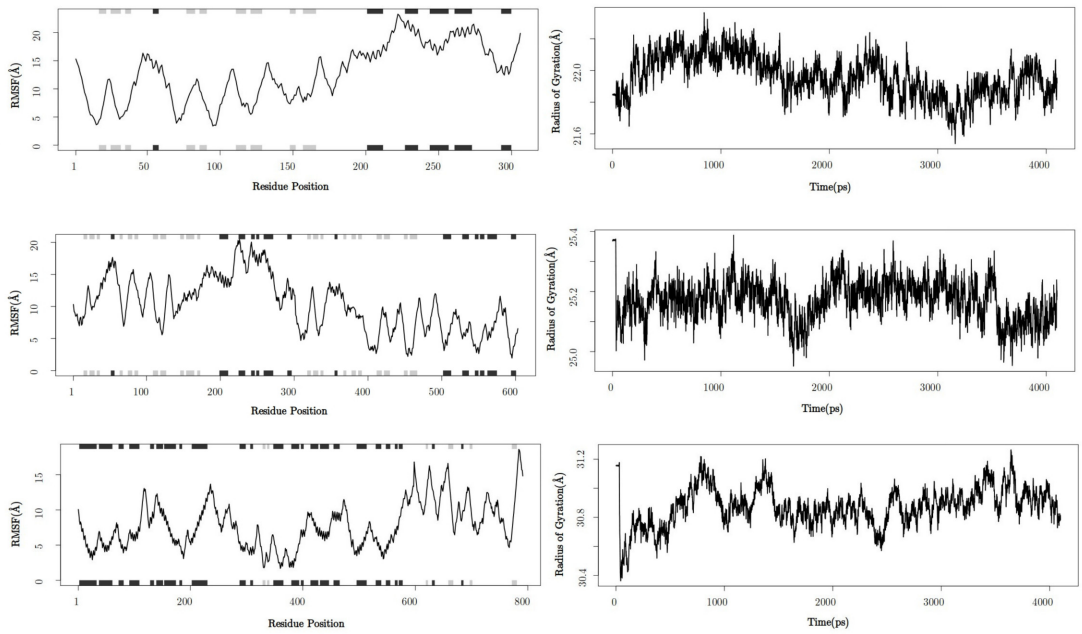

Figure 12: Molecular dynamics analysis of proteins SARS-CoV-2 Mpro, SARS-CoV 3CLpro, ACE2 Receptor involved in disease COVID-19 in complex with chloroquine.

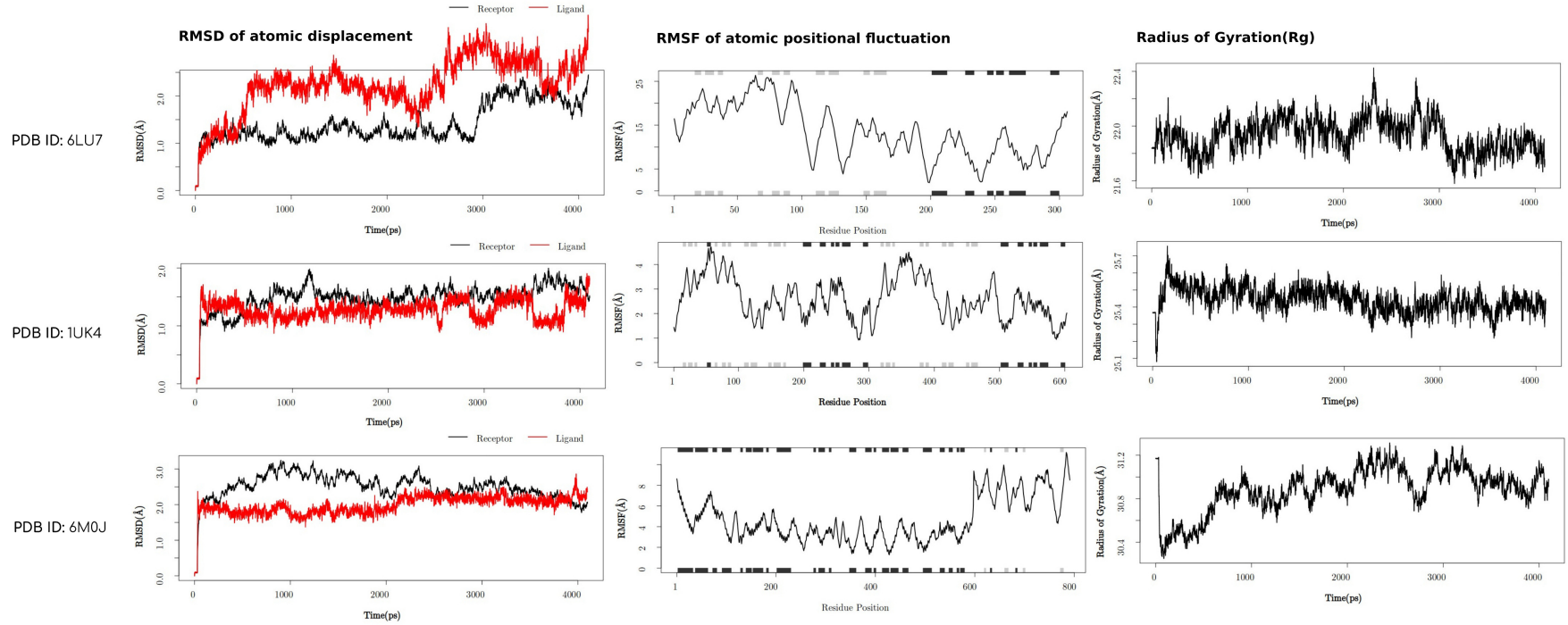

Figure 13: Molecular dynamics analysis of proteins SARS-CoV-2 Mpro, SARS-CoV 3CLpro, ACE2 Receptor involved in disease COVID-19 in complex with simeprevir. 


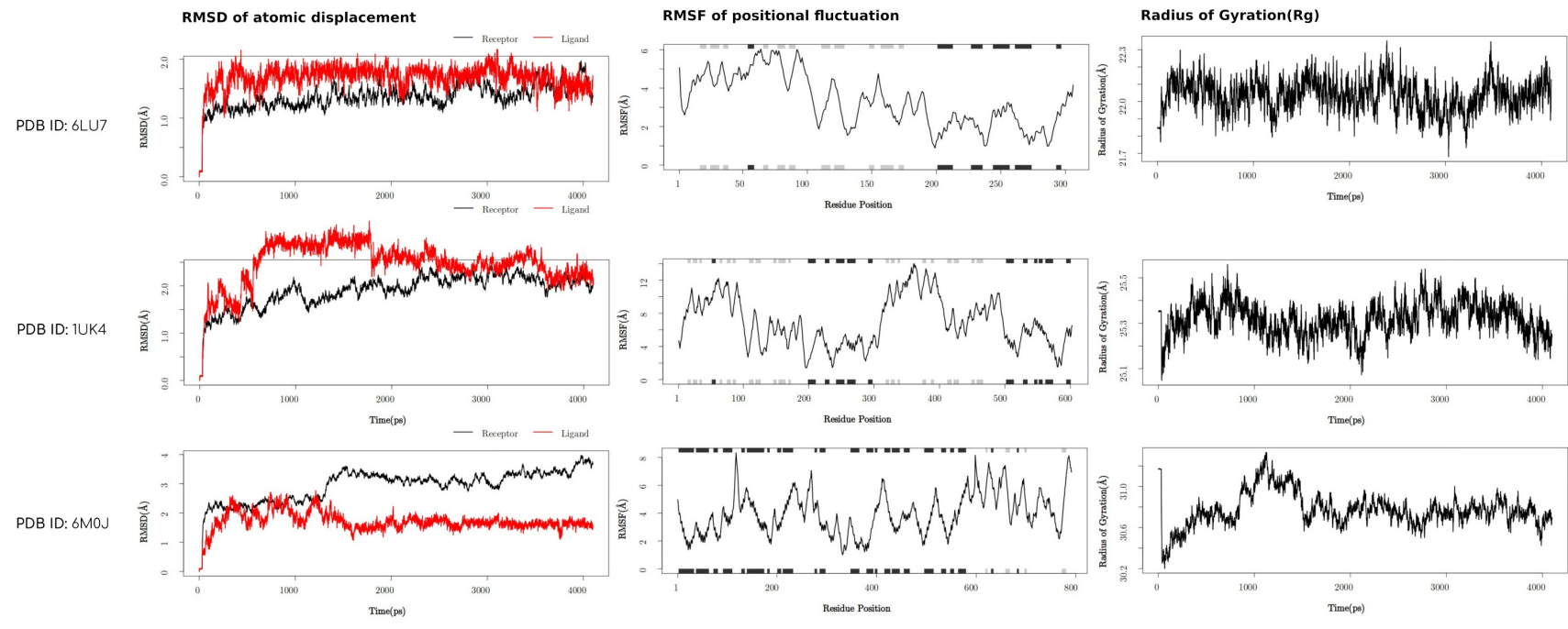

Figure 14: Molecular dynamics analysis of proteins SARS-CoV-2 Mpro, SARS-CoV 3CLpro, ACE2 Receptor involved in disease COVID-19 in complex with paritaprevir.

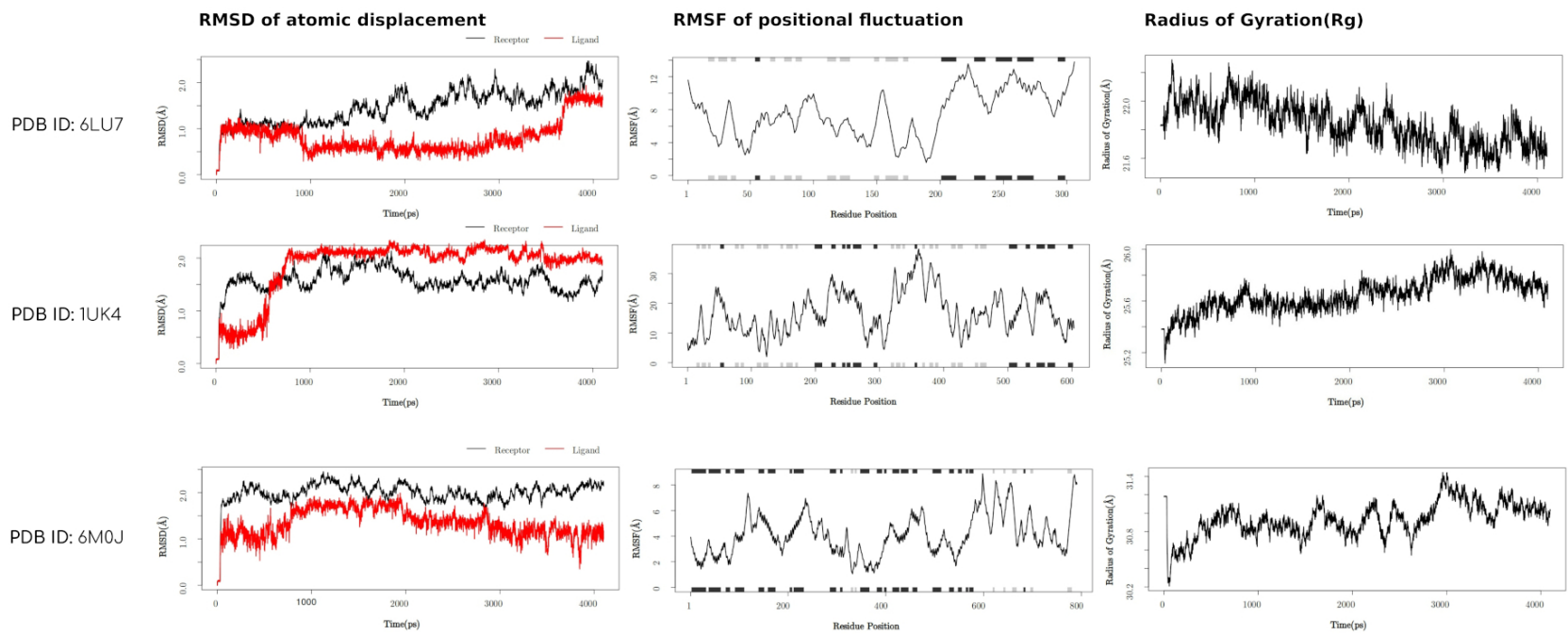

Figure 15: Molecular dynamics analysis of proteins SARS-CoV-2 Mpro, SARS-CoV 3CLpro, ACE2 Receptor involved in disease COVID-19 in complex with bariticinib.

All results were made with $L A R M D$ tool at a $A m$ ber16 force field [62]. In the ligands that cause major conformational changes in the receptor, it is noticed that the probability of a higher fluctuation in the RMSD, RMSF and Rg graphs. The estimation of free energy takes into account electrostatic energy $\left(\Delta E_{V D W}+\Delta G_{P B}\right)$, Van der Waals contribution $\left(\Delta E_{V D W}\right)$, total gas phase energy (GAS), non-polar and polar contributions to solvation $\left(\Delta G_{S A}\right)$ and entropic state $(T \Delta S)$. In the RMSF charts, helices are highlighted in black, $\beta$ strands in gray and loops in white (see Figures 13-16).
Larger fluctuations are expected in the loop regions.

The temporal evolution in the RMSD and RMSF graphs shows that in fact the simeprevir and paritaprevir structures cause high flexibility in the receptors. In contrast, chloroquine causes less conformational mobility of the receptor compared to more promising drugs. Even after the 4ns time elapsed, it was not possible to reach an equilibrium state in the complexes, indicating that longer simulations could reveal more details about the mechanism of action of the drugs. Thus, the molecular dynamics simulations corroborate the par- 
Table 6

$\mathrm{MM} / \mathrm{PB}(\mathrm{GB}) \mathrm{SA}$ binding free energies (in $\mathrm{kcal} / \mathrm{mol}$ ) of some ligands in complex with SARS-CoV-2 Mpro. The entropic contribution was estimated using $T \approx 298.15 K$.

\begin{tabular}{llllll}
\hline Antagonist & $\Delta E_{V D W}$ & $\Delta E_{V D W}+\Delta G_{P B}$ & $\Delta G_{P B}$ & $\Delta G_{S A}$ & $T \Delta S$ \\
\hline Simeprevir & -41.73 & -4.51 & -13.27 & $19.34 / 19.27$ & 13.62 \\
Paritaprevir & -48.38 & -18.20 & -18.28 & $35.65 / 30.44$ & 12.65 \\
Bariticinib & -28.46 & -44.22 & -8.83 & $54.52 / 52.55$ & 9.33 \\
Chloroquine & -28.46 & -44.22 & -8.83 & $54.52 / 52.55$ & 9.33 \\
\hline
\end{tabular}

tial inhibition potential of the receptors involved in the replication of the SARS-CoV-2 virus. As well, there is a direct correlation between lower values of free energy and lower potential of the equilibrium state of the system, that is, greater potential for modulation, increasing the probability of higher RMSD in the simulations.

Previous studies have shown that although chloroquine has therapeutic activity against SARS-CoV in cell culture, but the cell surface levels of ACE2 remain unchanged [77]. However, the molecular dynamics of the chloroquine in complex with ACE2 still showed conformational mobility of the protein, this is because theoretical simulations do not take into account others possible biological factors. By calculations of $M M / P B(G B) S A$ the value of free energy in the chloroquine complex and the protein $6 L U^{\gamma}$, acquires the value of deltaPB $\approx$ $-8.83 \mathrm{kcal} / \mathrm{mol}$ (Table 6 ). In contrast, the ligand simeprevir presented deltaPB$\approx-13.62 \mathrm{kcal} / \mathrm{mol}$. Meanwhile, the paritaprevir structure has the value of $\operatorname{delta} P B \approx$ $-18.28 \mathrm{kcal} / \mathrm{mol}$. This is consistent with the docking results, in which simeprevir and paritaprevir also had the lowest values of free energy.

Finally, the effects of conformational factors were investigated through principal component analysis (PCA). PCA can shows important information about the nature of conformational differences involved in proteins. The PCA results for trajectory have blue to red colored framesover time and represent the two states for a protein: active and inactive, respectively. All stages of protein folding can be identified. These calculations for the drugs simeprevir, paritaprevir, chloroquine and bariticinib, are showed in (Figure 16). All four drugs responded well, but note that the insertion of paritaprevir results in a greater degree of inactivity of the SARS-CoV-2 main protease, followed by simeprevir. On the other hand, comparativelly this protein showed a milder dynamic response in the presence of chloroquine.
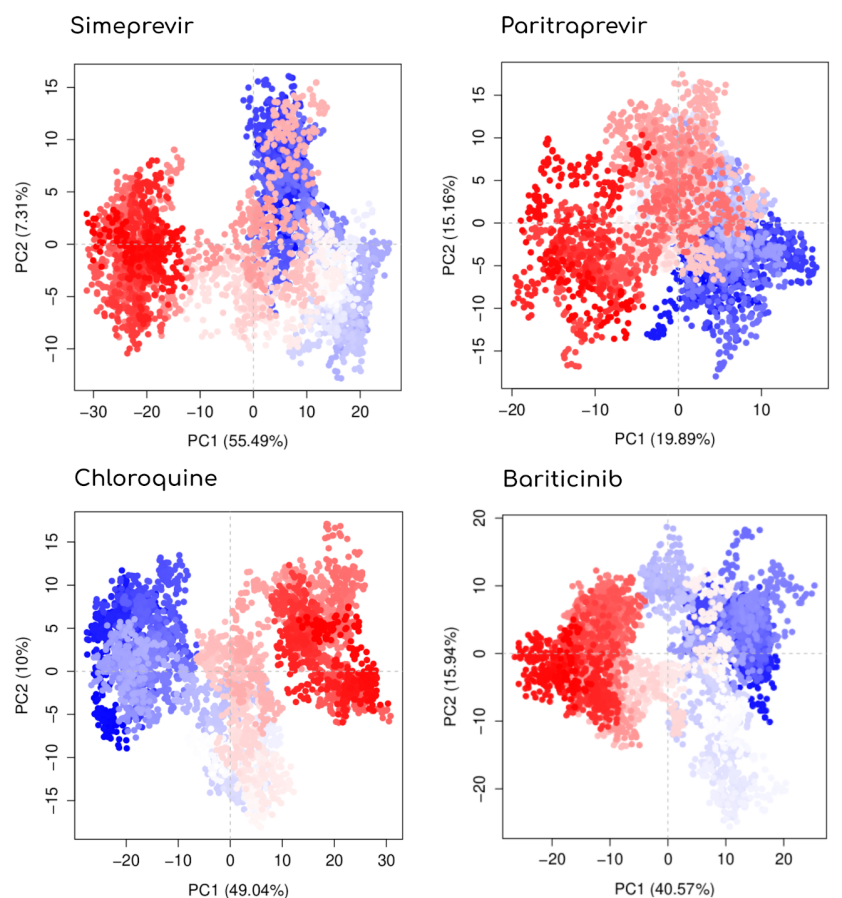

Bariticinib

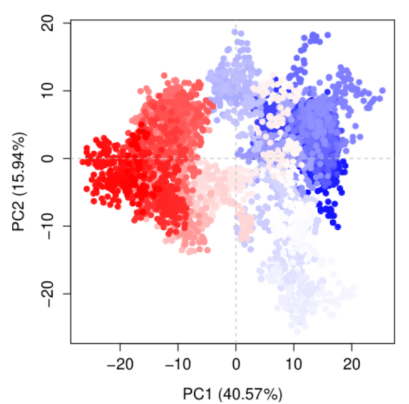

Figure 16: PCA results trajectories for complexes of ligands with SARS-CoV-2 Mpro. 


\section{Conclusions}

This work sought to analyze and compare, from various theoretical approaches, the properties of 28 drugs used in different diseases, which reported as potential antagonists against COVID-19. Chloroquine, (hydroxy)chloroquine, chloroquine phosphate and favipiravir, that are in great evidence at the present moment, has been shown to have a low affinity for interaction with major virus receptors. Not corroborating some important clinical results, indicating they are not effective, at least, as monotherapy. Although docking results, all these drugs have excellent pharmacokinetic and physical-chemical properties, there was a higher absorption with oral administration, leading to remission of symptoms in specific clinic cases reported in literature.

On the other hand, remdesivir, the drug widely studied by researchers around the world, has shown itself to be extremely promising in acting as antagonist of the main receptors involved in the viral cycle. Although the docking and molecular dynamics simulations have their limitations, and the flexibility of the receptor has not been taken into account, nor even a previous structural optimization of the ligands, it seems, the mean of $\overline{\Delta G} \approx-8.125 \mathrm{kcal} / \mathrm{mol}$ shows a promising drug. The molecular dynamics simulations indicates a significant unfolding of all receptors compared to chloroquine and its similars. This corroborates the hypothesis that the main mechanism of aminoquinolines is not to interact with the virus receptors, but to increase the intracellular $\mathrm{pH}$ and prevent passage through the phospholipid membrane and the consequent release of the viral genome inside the cell for interaction of the SARS-CoV2 (S) glycoprotein with the ACE-2 receptors.

Azithromicin, despite being an antibiotic, has theoretically shown to be as effective as remdemsivir. This corroborates experimental studies, in addition to in- dicating that azithromicin interacts remarkably with the receptors involved in the replication of the virus. In part, this can be explained by the higher reactivity among all 6 drugs that had their electronic descriptors analyzed. On the other hand, it is more susceptible to adverse effects.

It is worth mentioning 2 important drugs in the treatment of patients affected by the Hepatitis-C virus, simeprevir and paritaprevir, proved to be crucial candidates for the treatment of patients in the most serious condition with COVID-19. Presenting an average free energy of inhibition, respectively, $\overline{\Delta G} \approx-9.675 \mathrm{kcal} / \mathrm{mol}$ and $\overline{\Delta G} \approx-9.825 \mathrm{kcal} / \mathrm{mol}$ with excellent RMSD values obtained by superimposing the input structure and the best conformer, present mean values of $R M S D_{U B} \approx$ $1.638 \AA$ and $R M S D_{U B} \approx 1.697 \AA$. Corroborating the theoretical results obtained by Alamri et al. with the same ligands. Indinavir should be praised as having a medically potential remission in more severe cases of the disease, whose $\overline{\Delta G} \approx-9.475$.

However, few experimental researches has yet been done with simeprevir, paritaprevir and elbasvir, thus the results of this research cannot be conclusive. Because theoretical results have many limitations, and moreover, many questions still surround viral mechanism. This research sought from broad theoretical approach, understand the inhibition potential of the most reported drugs in the literature in the treatment of COVID-19. In this way, the results obtained aim to direct the research to certain ligands so that other researchers can carry out experimental tests in the hope of finally eradicating this disease and saving many patients.

\section{Conflicts of interests}

The authors declare no conflict of interest. 


\section{Acknowledgements}

We express our gratitude to all researchers and people who came together to help each other in the fight against the SARS-CoV-2 virus. We are also grateful to the Brazilian agency FAPEAM for supporting this research.

\section{References}

[1] Q. Li, X. Guan, P. Wu, X. Wang, L. Zhou, Y. Tong, et al., Early Transmission Dynamics in Wuhan, China, of Novel Coronavirus-Infected Pneumonia, New England Journal of Medicine 382 (13) (2020) 1199-1207. doi:10.1056/ NEJMoa2001316.

[2] K. Shen, Y. Yang, et al., Diagnosis, treatment, and prevention of 2019 novel coronavirus infection in children: experts' consensus statement, World Journal of Pediatrics (2020). doi:10.1007/s12519-020-00343-7.

[3] World Health Organisation, Coronavirus disease 2019 (covid-19) situation report-83, https://www.who.int/ emergencies/diseases/novel-coronavirus-2019/situation-reports, (acessed on April 12, 2020).

[4] J. Cui, F. Li, Z.-L. Shi, Origin and evolution of pathogenic coronaviruses, Nature Reviews Microbiology 17 (3) (2019) 181 - 192. doi:10.1038/s41579-018-0118-9.

[5] C. Huang, Y. Wang, X. Li, L. Ren, J. Zhao, Y. Hu, et al., Clinical features of patients infected with 2019 novel coronavirus in Wuhan, China, The Lancet 395 (10223) (2020) 497-506. doi:10.1016/s0140-6736(20)30183-5.

[6] P. Zhou, X.-L. Yang, X.-G. Wang, B. Hu, L. Zhang, W. Zhang, et al., A pneumonia outbreak associated with a new coronavirus of probable bat origin, Nature 579 (7798) (2020) 507 - 513. doi:10.1038/s41586-020-2012-7.

[7] C. Rothe, M. Schunk, P. Sothmann, G. Bretzel, G. Froeschl, C. Wallrauch, et al., Transmission of 2019-nCoV Infection from an Asymptomatic Contact in Germany, New England Journal of Medicine 382 (10) (2020) 970-971. doi:10.1056/ NEJMC2001468.

[8] E. de Wit, N. van Doremalen, D. Falzarano, V. J. Munster, SARS and MERS: recent insights into emerging coronaviruses, Nature Reviews Microbiology 14 (8) (2016) 523524. doi:10.1038/nrmicro.2016.81.

[9] T. M. Nguyen, Y. Zhang, P. P. Pandolfi, Virus against virus: a potential treatment for 2019-nCov (SARS-CoV-2) and other RNA viruses, Cell Research 30 (3) (2020) 189-190. doi: 10.1038/s41422-020-0290-0.

[10] T. Wang, Z. Du, F. Zhu, Z. Cao, Y. An, Y. Gao, B. Jiang, Comorbidities and multi-organ injuries in the treatment of COVID-19, The Lancet 395 (10228) (2020) 52. doi:10.1016/ S0140-6736(20)30558-4.

[11] N. Chen, M. Zhou, X. Dong, J. Qu, F. Gong, Y. Han, et al., Epidemiological and clinical characteristics of 99 cases of 2019 novel coronavirus pneumonia in Wuhan, China: a descriptive study, The Lancet 395 (10223) (2020) 507 - 513. doi:10.1016/S0140-6736(20)30211-7.

[12] Y. Shi, Y. Wang, C. Shao, J. Huang, J. Gan, X. Huang, et al., COVID-19 infection: the perspectives on immune responses, Cell Death \& Differentiation (2016). doi:10.1038/ s41418-020-0530-3.

[13] X. Ou, Y. Liu, X. Lei, P. Li, D. Mi, L. Ren, et al., Characterization of spike glycoprotein of SARS-CoV-2 on virus entry and its immune cross-reactivity with SARSCoV, Nature Communications 11 (1) (2020). doi:10.1038/ s41467-020-15562-9.

[14] M. Macchiagodena, M. Pagliai, P. Procacci, Inhibition of the Main Protease 3CL Pro of the Coronavirus Disease 19 via Structure-Based Ligand Design and Molecular Modeling (2020).

[15] P. Sang, S. Tian, Z. Meng, L. Yang, Insight Derived from Molecular Docking and Molecular Dynamics Simulations into the Binding Interactions Between HIV-1 Protease Inhibitors and SARS-CoV-2 3CLpro (2020). doi:10.26434/ chemrxiv.11932995.v1.

[16] Y.-W. Kam, Y. Okumura, H. Kido, L. F. P. Ng, R. Bruzzone, R. Altmeyer, Cleavage of the SARS Coronavirus Spike Glycoprotein by Airway Proteases Enhances Virus Entry into Human Bronchial Epithelial Cells In Vitro, Public Library of Science 4 (11) (2009) 1-10. doi:10.1371/journal. pone. 0007870.

[17] B. Robson, Computers and viral diseases. Preliminary bioinformatics studies on the design of a synthetic vaccine and a preventative peptidomimetic antagonist against the SARSCoV-2 (2019-nCoV, COVID-19) coronavirus, Computers in Biology and Medicine (2020). doi:10.1016/j.compbiomed. 2020. 103670.

[18] Y. Chen, Q. Liu, D. Guo, Emerging coronaviruses: Genome structure, replication, and pathogenesis, Journal of Medical Virology 92 (4) (2020) 418-423. doi:10.1002/jmv. 25681.

[19] D. Wrapp, N. Wang, K. S. Corbett, J. A. Goldsmith, C.-L. Hsieh, O. Abiona, B. S. Graham, J. S. McLellan, 
Cryo-EM structure of the 2019-nCoV spike in the prefusion conformation, Science 367 (6483) (2020) 1260-1263. doi:10.1126/science. abb2507.

[20] A. C. Walls, Y.-J. Park, M. A. Tortorici, A. Wall, A. T. McGuire, D. Veesler, Structure, Function, and Antigenicity of the SARS-CoV-2 Spike Glycoprotein, Cell (2020). doi: $10.1016 / j . c e l 1.2020 .02 .058$.

[21] H. Zhang, J. M. Penninger, Y. Li, N. Zhong, A. S. Slutsky, Angiotensin-converting enzyme 2 (ACE2) as a SARS-CoV-2 receptor: molecular mechanisms and potential therapeutic target, Intensive Care Medicine (2020) 586-590doi:10.1007/ s00134-020-05985-9.

[22] F. Jensen, Rang \& Dale: Pharmacology, 9th Edition, Elsevier, 2020, p. 296.

[23] C. Wu, Y. Liu, Y. Yang, P. Zhang, W. Zhong, Y. Wang, Q. Wang, et al., Analysis of therapeutic targets for SARS$\mathrm{CoV}-2$ and discovery of potential drugs by computational methods, Acta Pharmaceutica Sinica B (2020). doi:10.1016/ j.apsb.2020.02.008.

[24] B. H. Harcourt, D. Jukneliene, A. Kanjanahaluethai, J. Bechill, K. M. Severson, C. M. Smith, et al., Identification of Severe Acute Respiratory Syndrome Coronavirus Replicase Products and Characterization of Papain-Like Protease Activity, Journal of Virology 78 (24) (2004) 13600-13612. doi:10.1128/JVI.78.24.13600-13612.2004.

[25] H. Yang, W. Xie, X. Xue, K. Yang, J. Ma, W. Liang, et al., Design of Wide-Spectrum Inhibitors Targeting Coronavirus Main Proteases, PLOS Biology 3 (10) (09 2005). doi:10.1371/ journal. pbio.0030324.

[26] J. S. Morse, T. Lalonde, S. Xu, W. Liu, Learning from the Past: Possible Urgent Prevention and Treatment Options for Severe Acute Respiratory Infections Caused by 2019nCoV, ChemBioChem 21 (5) (2020) 730-738. doi:10.1002/ cbic. 202000047.

[27] L. Subissi, I. Imbert, F. Ferron, A. Collet, B. Coutard, E. Decroly, et al., SARS-CoV ORF1b-encoded nonstructural proteins 12-16: Replicative enzymes as antiviral targets, Antiviral Research 101 (2014) 122-130. doi:10.1016/j. antiviral.2013.11.006.

[28] J. Wang, Fast Identification of Possible Drug Treatment of Coronavirus Disease -19 (COVID-19) Through Computational Drug Repurposing Study (2020). doi : 10.26434/chemrxiv. 11875446.v1.

[29] M. A. Alamri, M. T. ul Qamar, S. M. Alqahtani, Pharmacoinformatics and molecular dynamic simulation studies reveal potential inhibitors of SARS-CoV-2 main protease 3CL pro (2020). doi:10.20944/preprints202002.0308.v1.

[30] Y.-C. Chang, Y.-A. Tung, K.-H. Lee, T.-F. Chen, Y.-C. Hsiao, H.-C. Chang, et al., Potential therapeutic agents for COVID-19 based on the analysis of protease and RNA polymerase docking (2020). doi:10.20944/preprints202002.0242.v1.

[31] T. P. Sheahan, A. C. Sims, S. R. Leist, A. Schäfer, J. Won, A. J. Brown, et al., Comparative therapeutic efficacy of remdesivir and combination lopinavir, ritonavir, and interferon beta against MERS-CoV, Nature Communications 11 (2020). doi:10.1038/s41467-019-13940-6.

[32] M. Wang, R. Cao, L. Zhang, X. Yang, J. Liu, M. Xu, et al., Remdesivir and chloroquine effectively inhibit the recently emerged novel coronavirus (2019-nCoV) in vitro, Cell Research 30 (2020) 269-271. doi:10.1038/s41422-020-0282-0.

[33] S. Wan, Y. Xiang, W. Fang, Y. Zheng, B. Li, Y. Hu, et al., Clinical Features and Treatment of COVID-19 Patients in Northeast Chongqing, Journal of Medical Virology (2020). doi: $10.1002 / j m v .25783$.

[34] P. Gautret, J.-C. Lagier, P. Parola, V. T. Hoang, L. Meddeb, M. Mailhe, et al., Hydroxychloroquine and azithromycin as a treatment of COVID-19: results of an open-label nonrandomized clinical trial, International Journal of Antimicrobial Agents (2020). doi:10.1016/j.ijantimicag. 2020.105949.

[35] L. Dong, S. Hu, J. Gao, Discovering drugs to treat coronavirus disease 2019 (COVID-19), Drug Discoveries \& Therapeutics 14 (1) (2020) 58-60. doi:10.5582/ddt.2020.01012.

[36] A. A. Elfiky, Anti-HCV, nucleotide inhibitors, repurposing against COVID-19, Life Sciences 248 (2020) 117477. doi: 10.1016/j.lfs. 2020.117477.

[37] C. Gruber, G. Steinkellner, Wuhan coronavirus 2019$\mathrm{nCoV}$ - what we can find out on a structural bioinformatics level, https://innophore.com/2019-ncov/, (acessed on March 21, 2020).

[38] P. Richardson, I. Griffin, C. Tucker, D. Smith, O. Oechsle, A. Phelan, J. Stebbing, Baricitinib as potential treatment for 2019-nCoV acute respiratory disease, The Lancet 395 (10223) (2020) 30-31. doi:10.1016/S0140-6736(20)30304-4.

[39] X. Liu, B. Zhang, Z. Jin, H. Yang, Z. Rao, et al., Structure of Mpro from COVID-19 virus and discovery of its inhibitors (2020). doi:10.1101/2020.02.26.964882.

[40] H. Yang, M. Yang, Y. Ding, Y. Liu, Z. Lou, Z. Zhou, L. Sun, et al., The crystal structures of severe acute respiratory syndrome virus main protease and its complex with an inhibitor, Proceedings of the National Academy of Sciences 100 (23) (2003) 13190-13195. doi:10.1073/pnas.1835675100.

[41] X. Wang, J. Lan, J. Ge, J. Yu, S. Shan, et al., Crystal struc- 
ture of 2019-nCoV spike receptor-binding domain bound with ACE2, Nature (2020). doi:10.1038/s41586-020-2180-5.

[42] R. N. Kirchdoerfer, A. B. Ward, Structure of the SARSCoV nsp12 polymerase bound to nsp7 and nsp8 cofactors, Nature Communications 10 (1) (2019). doi:10.1038/ s41467-019-10280-3.

[43] L. A. Kelley, S. Mezulis, C. M. Yates, M. N. Wass, M. J. E. Sternberg, The Phyre2 web portal for protein modeling, prediction and analysis, Nature Protocols 10 (6) (2015) 845858. doi:10.1038/nprot.2015.053.

[44] D. L. Nelson, M. M. Cox, Lehninger Principles of Biochemistry, 7th Edition, Macmillan Higher Education, 2017, p. 119.

[45] S. C. Lovell, I. W. Davis, W. B. Arendall III, P. I. W. de Bakker, J. M. Word, M. G. Prisant, et al., Structure validation by $\mathrm{c} \alpha$ geometry: $\phi, \psi$ and $\mathrm{c} \beta$ deviation, Proteins: Structure, Function, and Bioinformatics 50 (3) (2003) 437450. doi:10.1002/prot. 10286.

[46] A. Daina, O. Michielin, V. Zoete, SwissADME: a free web tool to evaluate pharmacokinetics, drug-likeness and medicinal chemistry friendliness of small molecules, Scientific Reports 7 (1) (2017). doi:10.1038/srep42717.

[47] P. D. Leeson, B. Springthorpe, The influence of drug-like concepts on decision-making in medicinal chemistry, Nature Reviews Drug Discovery 6 (11) (2007) 881-890. doi:10.1038/ $\operatorname{nrd} 2445$.

[48] L. L. Brunton, R. Hilal-Dandan, B. C. Knollmann, Goodman \& Gilman's: The pharmacological basis of therapeutics, McGraw-Hill Education, 2018, p. 7.

[49] O. Trott, A. J. Olson, AutoDock Vina: Improving the speed and accuracy of docking with a new scoring function, efficient optimization, and multithreading, Journal of Computational Chemistry 31 (2) (2010) 455-461. doi: 10.1002/jcc. 21334 .

[50] J. Nocedal, S. Wright, Numerical Optimization, 1st Edition, Springer-Verlag, Berlin, 1999.

[51] B. D. Bursulaya, M. Totrov, R. Abagyan, C. L. B. III, Comparative study of several algorithms for flexible ligand docking, Journal of computer-aided molecular design 17 (11) (2003) 755-763. doi:10.1023/B: JCAM.0000017496.76572.6f.

[52] E. F. Pettersen, T. D. Goddard, C. C. Huang, G. S. Couch, D. M. Greenblatt, E. C. Meng, et al., UCSF Chimera-A visualization system for exploratory research and analysis, Journal of Computational Chemistry 25 (13) (2004). doi: 10.1002/jcc. 20084.

[53] M. Sanner, Python: a programming language for software integration and development, Journal of molecular graphics \& modelling 17 (1) (1999) 57-61.

[54] J. Wang, W. Wang, P. A. Kollman, D. A. Case, Automatic atom type and bond type perception in molecular mechanical calculations, Journal of Molecular Graphics and Modelling 25 (2) (2006) 247-260. doi:10.1016/j.jmgm.2005.12.005.

[55] G. M. Morris, D. S. Goodsell, R. S. Halliday, R. Huey, W. E. Hart, R. K. Belew, A. J. Olson, Automated docking using a Lamarckian genetic algorithm and an empirical binding free energy function, Journal of Computational Chemistry 19 (14) (1998) 1639-1662. doi:10.1002/ (SICI) 1096-987X (19981115) 19:14<1639: : AID-JCC10>3 . 0. C0;2-B.

[56] N. S. Pagadala, K. Syed, J. Tuszynski, Software for molecular docking: a review, Biophysical Reviews 9 (2) (2017) 91-102. doi:10.1007/s12551-016-0247-1.

[57] A. Fersht, Structure and mechanism in protein science: a guide to enzyme catalysis and protein folding, 2nd Edition, W. H. Freeman and Company, 1998, p. 68.

[58] A. Carr, D. A. Cooper, Open Babel: An open chemical toolbox, Journal of Cheminformatics 3 (33) (2011). doi: $10.1186 / 1758-2946-3-33$.

[59] M. W. Schmidt, K. K. Baldridge, J. A. Boatz, S. T. Elbert, M. S. Gordon, J. H. Jensen, et al., General atomic and molecular electronic structure system, Journal of Computational Chemistry 14 (11) (1993) 1347-1363. doi:10.1002/ jcc. 540141112.

[60] J. Schmidt, W. Polik, WebMO Enterprise Version 19.0.009e, https://www. webmo. net/demoserver/cgi-bin/webmo/login. cgi, (acessed on March 27, 2020).

[61] A. R. Leach, Molecular modelling principles and applications, 2nd Edition, Pearson Education Limited, 2001, p. 353.

[62] J.-F. Yang, F. Wang, Y.-Z. Chen, G.-F. Hao, G.-F. Yang, LARMD: integration of bioinformatic resources to profile ligand-driven protein dynamics with a case on the activation of estrogen receptor, Briefings in Bioinformatics (2019). doi: 10.1093/bib/bbz141.

[63] D. A. Case, T. E. Cheatham III, T. Darden, H. Gohlke, R. Luo, K. M. Merz Jr., et al., The Amber biomolecular simulation programs, Journal of Computational Chemistry 26 (16) (2005) 1668-1688. doi:10.1002/jcc. 20290.

[64] S. Tian, Z. Meng, L. Yang, Insight Derived from Molecular Docking and Molecular Dynamics Simulations into the Binding Interactions Between HIV-1 Protease Inhibitors and SARS-CoV-2 3CLpro (2020). doi:https://10.26434/ chemrxiv.11932995.v1. 
[65] G. de Albuquerque Barros, Aplicação de docking molecular em estudos de inibição enzimática in silico: um estudo comparativo, Undergraduate Completed Monography. Orient. K.M.T. Oliveira, Manaus (2019).

[66] Z. Wang, H. Sun, X. Yao, D. Li, L. Xu, Y. Li, et al., Comprehensive evaluation of ten docking programs on a diverse set of protein-ligand complexes: the prediction accuracy of sampling power and scoring power, Physical Chemistry Chemical Physics 18 (2016) 12964-12975. doi:10.1039/ C6CP01555G.

[67] D. Plewczynski, M. Łaźniewski, R. Augustyniak, K. Ginalski, Can we trust docking results? Evaluation of seven commonly used programs on PDBbind database, Journal of Computational Chemistry 32 (4) (2011) 742-755. doi: 10.1002/jcc. 21643.

[68] B. Cao, Y. Wang, D. Wen, W. Liu, J. Wang, G. Fan, et al., A Trial of Lopinavir-Ritonavir in Adults Hospitalized with Severe Covid-19, New England Journal of Medicine (2020). doi : 10.1056/NEJMoa2001282.

[69] A. Cortegiani, G. Ingoglia, M. Ippolito, A. Giarratano, S. Einav, A systematic review on the efficacy and safety of chloroquine for the treatment of COVID-19, Journal of Critical Care (2020). doi:10.1016/j.jcrc.2020.03.005.

[70] M. de Abreu Silva, Modelos preditivos baseados em descritores moleculares e modos de interação receptor-ligante para inibidores de Acetilcolinesterase, Ph.D. thesis, Universidade de Brasília (2017).

[71] F. Jensen, Introduction to Computational Chemistry, 3rd Edition, John Wiley \& Sons, 2017, p. 515.

[72] K. Stierand, P. C. Maaß, M. Rarey, Molecular complexes at a glance: automated generation of two-dimensional complex diagrams, Bioinformatics 22 (14) (2006) 1710-1716. doi: 10.1093/bioinformatics/bt1150.

[73] B. Néron, H. Ménager, C. Maufrais, N. Joly, J. Maupetit, S. Letort, et al., Mobyle: a new full web bioinformatics framework, Bioinformatics 25 (22) (2009) 3005-3011. doi: 10.1093/bioinformatics/btp493.

[74] H. C. Jubb, A. P. Higueruelo, B. Ochoa-Montaño, W. R. Pitt, D. B. Ascher, T. L. Blundell, Arpeggio: A Web Server for Calculating and Visualising Interatomic Interactions in Protein Structures, Journal of Molecular Biology 429 (3) (2017) 365 - 371. doi:10.1016/j.jmb. 2016.12.004.

[75] X. Du, Y. Li, Y.-L. Xia, S.-M. Ai, J. Liang, P. Sang, et al., Insights into Protein-Ligand Interactions: Mechanisms, Models, and Methods, International journal of molecular sciences 17 (2) (2016). doi:10.3390/ijms17020144.
[76] H. Yogasundaram, B. N. Putko, J. Tien, D. I. Paterson, B. Cujec, J. Ringrose, et al., Hydroxychloroquine-Induced Cardiomyopathy: Case Report, Pathophysiology, Diagnosis, and Treatment, Canadian Journal of Cardiology 30 (12) (2014) 1706-1715. doi:10.1016/j.cjca.2014.08.016.

[77] M. J. Vincent, E. Bergeron, S. Benjannet, B. R. Erickson, P. E. Rollin, T. G. Ksiazek, et al., Chloroquine is a potent inhibitor of SARS coronavirus infection and spread, Virology Journal 2 (1) (2005). doi:10.1186/1743-422X-2-69. 


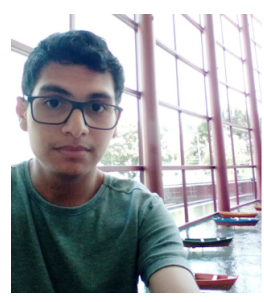

Micael D. L. Oliveira is a undergraduate student in Physics from the Federal University of Amazonas (2018). Has been working in docking research and computational modeling of biomolecules and promising drugs for the treatment of Major Depressive Disorder. Has experience in using Autodock Vina software for protein-ligand molecular docking using the Lamarckian genetic algorithm and via stochastic methods. In addition, acts in biomolecule dynamics via NAMD with CHARMM force field.

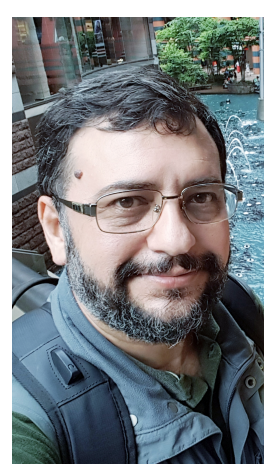

Kelson M. T. Oliveira earned bachelor's degree in Chemistry from the Federal University of Amazonas (1992), master's degree in Physics-Chemistry from the University of São Paulo (1996) and doctorate in PhysicsChemistry from the University of São Paulo (2000), researching in the area of Theoretical Chemistry / Molecular Modeling of the prosthetic hemoglobin nucleus. I was an associate professor at the Federal University of Rio Grande do Sul from 2007 to 2011, and currently returned to his duties as an Associate Professor at the Federal University of Amazonas. I have experience in the field of physical chemistry, with an emphasis on Theoretical Chemistry, acting mainly on the following topics: catalysts for fuel desulfurization; carbon nanostructures; metalloporphyrins for use in sensors and catalysts in fuel cells; theoretical modeling of natural products from the Amazonian flora aiming at activity against various parasitic disease and antineoplastics; synthetic routes of the fundamental blocks necessary for the appearance of life. Currently develops its activities in the Department of Chemistry / UFAM, in the Laboratory of Theoretical and Computational Chemistry. 